ACCEPTED FOR PUBLICATION IN THE Astronomical Journal.

Preprint typeset using $\mathrm{LT}_{\mathrm{E}} \mathrm{X}$ style emulateapj v. 08/22/09

\title{
ABSORPTION-LINE PROBES OF THE PREVALENCE AND PROPERTIES OF OUTFLOWS IN PRESENT-DAY STAR-FORMING GALAXIES
}

\author{
Yan-Mei Chen $^{1}$, Christy A. Tremonti ${ }^{2}$, Timothy M. Heckman ${ }^{3}$, Guinevere Kauffmann ${ }^{1}$, Benjamin J. Weiner ${ }^{4}$, Jarle $^{2}$ \\ BRINCHMANN $^{5}$, JING WANG ${ }^{1,6}$ \\ Accepted for publication in the Astronomical Journal.
}

\begin{abstract}
We analyze star forming galaxies drawn from SDSS DR7 to show how the interstellar medium (ISM) Na I $\lambda \lambda 5890,5896(\mathrm{Na} \mathrm{D})$ absorption lines depend on galaxy physical properties, and to look for evidence of galactic winds. We combine the spectra of galaxies with similar geometry/physical parameters to create composite spectra with signal-to-noise $\sim 300$. The stellar continuum is modeled using stellar population synthesis models, and the continuum-normalized spectrum is fit with two Na I absorption components. We find that: (1) ISM Na D absorption lines with equivalent widths EW $>0.8 \AA$ are only prevalent in disk galaxies with specific properties - large extinction $\left(\mathrm{A}_{\mathrm{V}}\right)$, high star formation rates (SFR), high star formation rate per unit area $\left(\Sigma_{\mathrm{SFR}}\right)$, or high stellar mass $\left(M_{*}\right)$. (2) the ISM Na D absorption lines can be separated into two components: a quiescent disk-like component at the galaxy systemic velocity and an outflow component; (3) the disk-like component is much stronger in the edge-on systems, and the outflow component covers a wide angle but is stronger within $60^{\circ}$ of the disk rotation axis; (4) the EW and covering factor of the disk component correlate strongly with dust attenuation, highlighting the importance that dust shielding may play in the survival of Na I. (5) The EW of the outflow component depends primarily on $\Sigma_{\mathrm{SFR}}$ and secondarily on $A_{V} ;(6)$ the outflow velocity varies from $\sim 120$ to $160 \mathrm{~km} \mathrm{~s}^{-1}$ but shows little hint of a correlation with galaxy physical properties over the modest dynamic range that our sample probes (1.2 dex in $\log \Sigma_{\text {SFR }}$ and 1 dex in $\left.\log M_{*}\right)$.
\end{abstract}

Subject headings: galaxies : evolution — galaxies : star formation

\section{INTRODUCTION}

Galactic-scale gaseous outflows ('galactic winds') are known to be ubiquitous in very actively star forming galaxies at all cosmic epochs (Heckman et al. 1990; Pettini et al. 2000; Shapley et al. 2003; Ménard et al. 2009; Weiner et al. 2009). Galactic winds play an vital role in the evolution of galaxies and the intergalactic medium (IGM). The "baryon deficit" in the Galaxy (e.g., Silk 2003) indicates gas removal by outflows during past active episodes. The massmetallicity and effective yield relations observed in local galaxies suggest that galactic winds transport highly metalenriched gas out of galaxies and into the IGM (e.g., Garnett 2002; Tremonti et al. 2004; Dalcanton 2007). The $\Lambda \mathrm{CDM}$ model over-predicts the galaxy luminosity function at both the low and high luminosity ends if a constant mass-to-light ratio is assumed. The most natural way to reconcile the observations with theory is to invoke feedback processes, including supernova, stellar winds (Cole et al. 2000; Benson et al. 2003; Stringer et al. 2009; Oppenheimer et al. 2009) and AGN activity (Silk \& Rees 1998; Hopkins et al. 2006). However, it remains unclear which kind of feedback processes dominate as a function of luminosity and comic epoch.

In actively star forming galaxies, galactic winds are

\footnotetext{
1 Max-Planck-Institut für Astrophysik, Karl-Schwarzschild-Str. 1, D85748 Garching, Germany

2 Department of Astronomy, University of Wisconsin-Madison, 1150 University Ave, Madison, WI 53706, USA

3 Department of Physics and Astronomy, The Johns Hopkins University, 3400 North Charles Street, Baltimore, MD 21218, USA

4 Steward Observatory, 933 N. Cherry St., University of Arizona, Tucson, AZ 85721, USA

5 Leiden Observatory, Leiden University, 2300 RA, Leiden, The Netherlands

6 Center for Astrophysics, University of Science and Technology of China, 230026 Hefei, China
}

driven by the mechanical energy and momentum imparted by stellar winds and supernovae (Chevalier \& Clegg 1985; Heckman et al. 1990). Young star clusters create overpressured bubbles of coronal phase gas which expand and sweep up shells of ambient ISM until they 'blow-out' of the disk into the halo. The collective action of multiple superbubbles drives a weakly collimated bi-polar outflow consisting of hot gas and cool entrained clouds. Radiation pressure is also likely to play a role in accelerating cool dusty material (e.g., Murray et al. 2005). In this paper we use the term 'galactic wind' to describe such outflows without regard for their eventual fate - the gas may escape the halo potential well, or be recycled back into the disk in a process sometimes referred to as 'galactic fountain' activity (Shapiro \& Field 1976; Bregman 1980; Kahn 1981).

In the last two decades, there have been many attempts to directly observe galactic winds in galaxies at $z=0-3$ (Veilleux et al. 2005). In the local universe, outflows can be detected via X-rays which trace hot gas (e.g., Dahlem et al. 1998; Martin et al. 2002; Strickland et al. 2004), optical nebular emission lines produced by warm gas (e.g., Lehnert \& Heckman 1996) and ISM absorption lines (e.g., Na I, K I) from cold gas (e.g., Heckman 2002). Blue-shifted Na D absorption from the entrained cool gas is frequently detected in IR-bright starburst galaxies (Heckman et al.|2000), LIRGs and ULIRGs (Rupke et al.2002, 2005a.b; Martin 2005, 2006; Martin \& Bouché 2009), and it is sometimes evident in dwarf starbursts (Schwartz \& Martin 2004). The velocity of this gas correlates weakly with star formation rate (SFR) and galactic rotation speed, with a factor of $\sim 30$ change in velocity observed over a range of 4 orders of magnitude in SFR $\left(v_{\text {wind }} \propto \mathrm{SFR}^{0.3} ;\right.$ Rupke et al. 2005b; Martin 2005).

At intermediate redshift, the Mg II $\lambda \lambda 2796,2803$ ISM absorption line shifts into the observed-frame optical. The 
relatively wide separation of this doublet makes it a good choice for outflow studies. Tremonti et al. (2007) detected $500-2000 \mathrm{~km} \mathrm{~s}^{-1}$ outflow velocities in a small sample of $z \sim 0.5$ post-starburst galaxies, and suggested that these outflows cold be the relics of AGN-driven winds. Weiner et al. (2009) employed the spectral stacking technique to probe $z \sim 1.4$ star forming galaxies drawn from the DEEP2 survey. This study, which is based on $\sim 1400$ galaxy spectra, demonstrated that blue-shifted Mg II absorption is ubiquitous in actively star forming galaxies.

At high redshift, the observed-frame optical samples the rest-frame far ultraviolet, which is rich in strong ISM resonance absorption transitions, but lacking in stellar spectral features. Because of the difficulty of measuring the relative velocity of the stars and gas, outflows have only been detected in very luminous Lyman break galaxies (Pettini et al. 2000, 2002) or in composite spectra (Shapley et al. 2003).

While much of our knowledge about galactic winds comes from studies of local star forming galaxies, the work to date has been based on relatively small samples of extreme objects (dwarf starbursts, ULIRGs). The properties of outflows in local normal star forming galaxies are still largely unknown. In this paper, we investigate $\mathrm{Na} \mathrm{D}$ absorption in a sample of $\sim 150,000$ star forming galaxies drawn from the Sloan Digital Sky Survey. By stacking the spectra of galaxies selected to have similar physical attributes, we obtain very high $\mathrm{S} / \mathrm{N}$ composite spectra. After carefully modeling and dividing out the stellar continuum, we are able to probe very low $\mathrm{Na} \mathrm{D}$ Equivalent Widths (EWs) and extend our analysis over a wide range in galaxy physical parameters.

This paper is arranged as follows. In $\S 2$, we introduce the sample selection criteria used in our study. Our method of creating composite spectra and measuring the $\mathrm{Na} \mathrm{D}$ lines is developed in $\$ 3$. We apply the method to the SDSS sample in $\S 4$ and summarize our results in $\$ 5$. We use the cosmological parameters $H_{0}=70 \mathrm{~km} \mathrm{~s}^{-1} \mathrm{Mpc}^{-1}, \Omega_{\mathrm{M}}=0.3$ and $\Omega_{\Lambda}=0.7$ throughout this paper.

\section{SAMPLE SELECTION}

\subsection{The Data}

The Sloan Digital Sky Survey (SDSS; York et al. 2000) spectroscopic galaxy catalogue contains $\sim 930,000$ spectra in its seventh data release (DR7; Abazaiian et al. 2009). We analyze objects drawn from the "Main" galaxy sample (Strauss et al. 2002), which have Petrosian $r$ magnitude in the range $14.5<r<17.7$ after correction for foreground galactic extinction using the reddening maps of Schlegel et al. (1998). The spectra are taken through $3^{\prime \prime}$ diameter fibers and cover a wavelength range from 3800 to $9100 \AA$ with a resolution of $R \sim 2000$ and a dispersion of $69 \mathrm{~km} \mathrm{~s}^{-1}$ pixel $^{-1}$. Most of the galaxies have redshifts in the range $z=0-0.3$. We adopt redshifts from the specBS pipeline (Adelman-McCarthy et al. 2008). The median redshift error is $\sim 10^{-4}$.

As described in Tremonti et al. (2004) and Brinchmann et al. (2004), a stellar population model is fit to the continuum of each galaxy spectrum after masking out the strong nebular emission lines. The basic assumption is that any galaxy star formation history can be approximated as a sum of discrete bursts. The library of template spectra is composed of single stellar population (SSP) models generated using a preliminary version of the population synthesis code of Charlot \& Bruzual (2010, in prep.) which incorporates the MILES empirical stellar library (Sánchez-Blázquez et al.
2006). We refer to these models hereafter as the 'CB08 models'. The spectral resolution (2.3 § FWHM), spectral-type coverage, flux-calibration accuracy and number of stars in the MILES library represent a substantial improvement over previous libraries used in population synthesis models. We generate template spectra with ten different ages $(0.005$, $0.025,0.1,0.2,0.6,0.9,1.4,2.5,5,10 \mathrm{Gyrs})$ and four metallicities $(0.004,0.008,0.017,0.04)$. For each metallicity, the ten template spectra are convolved to the measured stellar velocity dispersion of each individual SDSS galaxy, and the best fitting model spectrum is constructed from a non-negative linear combination of the template spectra, with dust attenuation modeled as an additional free parameter. The metallicity which yields the minimum $\chi^{2}$ is selected as the final best fit. The results of this fitting procedure and measurements of an number of line indices (e.g., Lick_Mgb, D4000) are made available in the MPA/JHU catalogue $\overline{7}^{7}$. The catalog also includes the equivalent width (EW) and flux of an number of emission and ISM absorption lines (e.g., $\mathrm{H} \alpha$, $\mathrm{H} \beta$, Na D).

The SDSS imaging data consist of CCD imaging in $u, g, r, i, z$ bands (Fukugita et al. 1996; Smith et al. 2002), taken with a drift scan camera (Gunn et al. 1998) mounted on a wide-field 2.5-m telescope. The SDSS photometric pipeline (Lupton et al. 2001) fits each galaxy image with a two-dimensional model of a de Vaucouleurs (1948) surface profile and an exponential profile, each convolved with the PSF of the image. A seeing-independent axial ratio $(b / a)$ is derived by this fitting procedure. The pipeline also computes the best linear combination of the exponential and de Vaucouleurs models and stores it in a parameter called fracDeV (Abazaiian et al. 2004). Here we follow Padilla \& Strauss (2008) in using fracDeV to distinguish disk galaxies (fracDeV $<0.8$ ) from early-type galaxies (fracDeV $\geq 0.8$ ). The axis ratios from the exponential and de Vaucouleurs models are consistent with each other independent of the morphology of the galaxy. In this work, we are only concerned with disk galaxies, and therefore we adopt the axis ratios from the exponential fit. We compute the galaxy inclination, $i$, from the measured axial ratio, $b / a$, and the $r$-band absolute magnitude $M_{\mathrm{r}}$ using Table 8 in Padilla \& Strauss (2008). This empirical relation takes into account the increase in bulge-to-disk ratio with absolute magnitude. Typical inclination errors are $10^{\circ}$ at $i>40^{\circ}$. At $i<40^{\circ}$ galaxy asymmetries can result in over-estimates of the inclination. The photometric properties used in this paper (fracDeV, $b / a, u, g, r, i, z$ magnitudes) are also available in catalogs on the MPA/JHU webpage.

The derived galaxy parameters required in this work include stellar mass $\left(M_{*}\right.$ for the whole galaxies and $M_{\text {fiber }}$ for stellar mass within fiber), stellar mass surface density $\left(\Sigma_{M_{*}}\right)$, $V$-band dust attenuation $\left(\mathrm{A}_{\mathrm{V}}\right)$, SFR, SFR surface density $\left(\Sigma_{\mathrm{SFR}}\right)$, and the SFR per unit stellar mass or 'specific SFR' $\left(\mathrm{SSFR}=\mathrm{SFR} / M_{\text {fiber }}\right)$. The stellar mass-to-light ratios are obtained by comparing urgiz colors of galaxies to a large grid of CB08 model colors following the methodology described in Salim et al. (2007) to avoid aperture correction issues. This approach differs from that of Kauffmann (2003a) who used the spectral indices $\mathrm{D}_{n}(4000)$ and $\mathrm{H} \delta_{\mathrm{A}}$ to constrain the massto-light ratios. However, the differences between the mass estimates of the two methods are very small, typically 0.01

\footnotetext{
$\begin{array}{ccc}7 & \text { The MPA/JHU catalog can be downloaded from } \\ \text { http://www.mpa-garching.mpg.de/SDSS/DR7 }\end{array}$
} 
dex.

We calculate $A_{V}$, the optical dust attenuation in magnitudes, using the $\mathrm{H} \alpha / \mathrm{H} \beta$ ratio. We assume an intrinsic $\mathrm{H} \alpha / \mathrm{H} \beta$ ratio of 2.87 (Osterbrock \& Ferland 2006), a Milky Way attenuation curve (Cardelli et al. 1989), and $R=3.1$. The nebular attuenation is likely to overestimate the attenuation of the stellar continuum (c.f., Calzetti et al. 1994).

We derive the SFR from the dust extinction corrected $\mathrm{H} \alpha$ luminosity using the formula of Kennicutt (1998a), dividing by a factor of 1.5 to convert from a Salpeter to Kroupa initial mass function (Kroupa 2001). $\Sigma_{\mathrm{SFR}}$ is defined as SFR $/ \pi R^{2}$ and $\Sigma_{\mathrm{M}_{*}}=M_{\text {fiber }} / \pi R^{2}$, where $R$ corresponds to the SDSS $1.5^{\prime \prime}$ aperture radius corrected for projection effects.

Except stellar mass, all of these derived parameters are calculated from quantities measured within the SDSS fiber aperture and we do not apply aperture corrections. We believe the locally determined physical parameters will have the greatest influence on the local ISM kinematics traced by $\mathrm{Na} \mathrm{D}$; star formation occurring in the outer disk of the galaxy is unlikely to drive a circum-nuclear outflow. Another exception to our policy of using locally derived physical properties is Figure 17 where we use global galaxy SFRs in order to compare with results from the literature. The global SFRs are estimated by adding the SFR inferred from the emission lines measured in the fiber aperture (Brinchmann et al. 2004) and the SFR estimated from the colors outside the fiber from fits to the 5-band photometry. The total SFRs are comparable the global SFRs in Brinchmann et al. (2004), but for a small subset of galaxies an aperture correction problem identified by Salim et al. (2007) has been rectified

\subsection{Sample Selection}

The criteria used to select the parent sample used in our analysis are the following:

1. redshift of $0.05 \leq \mathrm{z} \leq 0.18$. With $3^{\prime \prime}$ diameter fibers, the SDSS spectra probe the central 3-12 kpc in this redshift range. At the median redshift of $z=0.09$, the fiber probes the central $6 \mathrm{kpc}$.

2. $r$-band fracDeV $<0.8$. This ensures that we are selecting disk galaxies, which makes the calculation of the inclination angle from $b / a$ possible.

3. $\mathrm{D}_{n}(4000)<1.5$. This index measures the $4000 \AA$ break, and is a good indicator of the galaxy star formation history (Kauffmann 2003a). Our cut selects galaxies with young stellar populations. It is just below the threshold identified by Kauffmann (2003b, D $(4000)=1.55)$ where a sharp change in galaxy structural properties occurs.

4. $\log ([\mathrm{OIII}] / \mathrm{H} \beta)<0.61 /\{\log ([\mathrm{NII}] / \mathrm{H} \alpha)-0.05\}+1.3$. We exclude AGNs from our sample using the emission line ratio diagnostics given in Kauffmann (2003c).

We refer to this sample hereafter as 'Sample A'. It contains 140,625 galaxies. In $\$ 4$ we will justify the application of some additional cuts to help isolate galaxies with measurable interstellar $\mathrm{Na} \mathrm{D}$. In later sections of the paper, we will refer to these sub-samples as Samples B and C.

In Figure 1 we illustrate the distribution of galaxy physical properties in Sample A and show how these parameters correlate with one another. As we will discuss in $\$ 4.2$ these corre- lations make it difficult to distinguish the physical parameters that most strongly influence the properties of galactic winds.

\subsection{Interstellar Na D in SDSS Spectra}

Studying gas kinematics using optical absorption line spectroscopy is challenging because of the lack of strong ISM absorption lines. An additional complication when using the galaxy continuum as the background source, is the the prominence of stellar absorption features in the continuum. The $\mathrm{Na}$ I " $\mathrm{D}$ " $\lambda \lambda 5890,5896$ doublet is favored because it is moderately strong (sometimes saturated) in starburst galaxy spectra (c.f., Heckman et al. 2000), and because the stellar contamination is modest in galaxies dominated by very young stars. Other transitions which are sometimes used are $\mathrm{Ca}$ II $\mathrm{H} \&$ $\mathrm{K}$, which occur in a region of the spectrum rife with stellar absorption and nebular emission lines, and K I $\lambda \lambda 7665,7699$ which is a relatively weak transition.

The main drawback of using $\mathrm{Na} \mathrm{D}$ to probe the ISM of normal star forming galaxies is that it is a very prominent feature in the the spectra of cool stars; stellar contamination can therefore be significant. We discuss this issue further in $\$ 3.2$ where we show that $\sim 80 \%$ of the observed $\mathrm{Na} \mathrm{D}$ absorption in our galaxy spectra arises in stellar atmospheres.

In most previous work, the stellar contribution to the $\mathrm{Na}$ I line was estimated using the Mg I $\lambda \lambda 5167,5173,5184$ triplet, which arises only in stellar atmospheres. Martin (2005) find $\mathrm{EW}(\mathrm{Na} \mathrm{I})=1 / 3 \mathrm{EW}(\mathrm{Mg} \mathrm{I})$ based on an analysis of stellar spectra. Here we take a somewhat more sophisticated approach, and model the galaxy continuum light using stellar population synthesis models $(\$ 3.2)$. We use the Mg I lines residuals to gauge the success of our continuum fitting procedures.

In our subsequent analysis we will make use of composite galaxy spectra. However, we begin by examining individual spectra in Sample A with $\mathrm{S} / \mathrm{N}$ pixel $^{-1}>15$. Roughly $19 \%$ $(27,076)$ of the Sample A galaxies make this cut. For each galaxy we measure the $\mathrm{Na} \mathrm{D} \mathrm{EW}$ and the Lick $\mathrm{Mg} b$ index (Worthey et al. 1994) of the observed spectrum and the best fit continuum model. In Figure 2 we plot the residual absorption (data - model) as a function of nebular attenuation. We hypothesize that the $\mathrm{Na} \mathrm{D}$ excess in the data relative to the models is due to ISM absorption. Na I has an ionization potential less than Hydrogen, so dust shielding is expected to play an important role in its survival (Murray et al.2007). The strong positive correlation between the $\mathrm{Na} \mathrm{D}$ residual and dust attenuation is evidence that the excess $\mathrm{Na} \mathrm{D}$ absorption arises in the dusty ISM. The lack of any corresponding correlation in the $\mathrm{Mg} b$ residual indicates that the starlight is well fit by our models even in heavily dust attenuated galaxies. (The small positive offset of the median is due to the presence of a weak [N I] $\lambda 5198$ emission in one of the continuum bands of the Mg I index - see Fig. 5).

One notable feature of Figure 2 is tendency for galaxies with low dust attenuation to have negative $\mathrm{Na} \mathrm{D}$ residuals more $\mathrm{Na} \mathrm{D}$ absorption in the stellar population models than in the data. There are three possible explanations for this: (1) the $\mathrm{S} / \mathrm{N}$ of the spectra are not high enough for a robust continuum fit; (2) the stellar population models are not robust around the $\mathrm{Na} \mathrm{D}$ region, at least for some stellar populations; (3) $\mathrm{Na} \mathrm{D}$ is primarily in emission in gas and dust-poor galaxies. Through the spectral stacking technique, the $\mathrm{S} / \mathrm{N}$ issue can be easily resolved. We discuss the other possibilities further in $\$ 3.3$

\subsection{Galaxies with Strong ISM Na D Absorption}




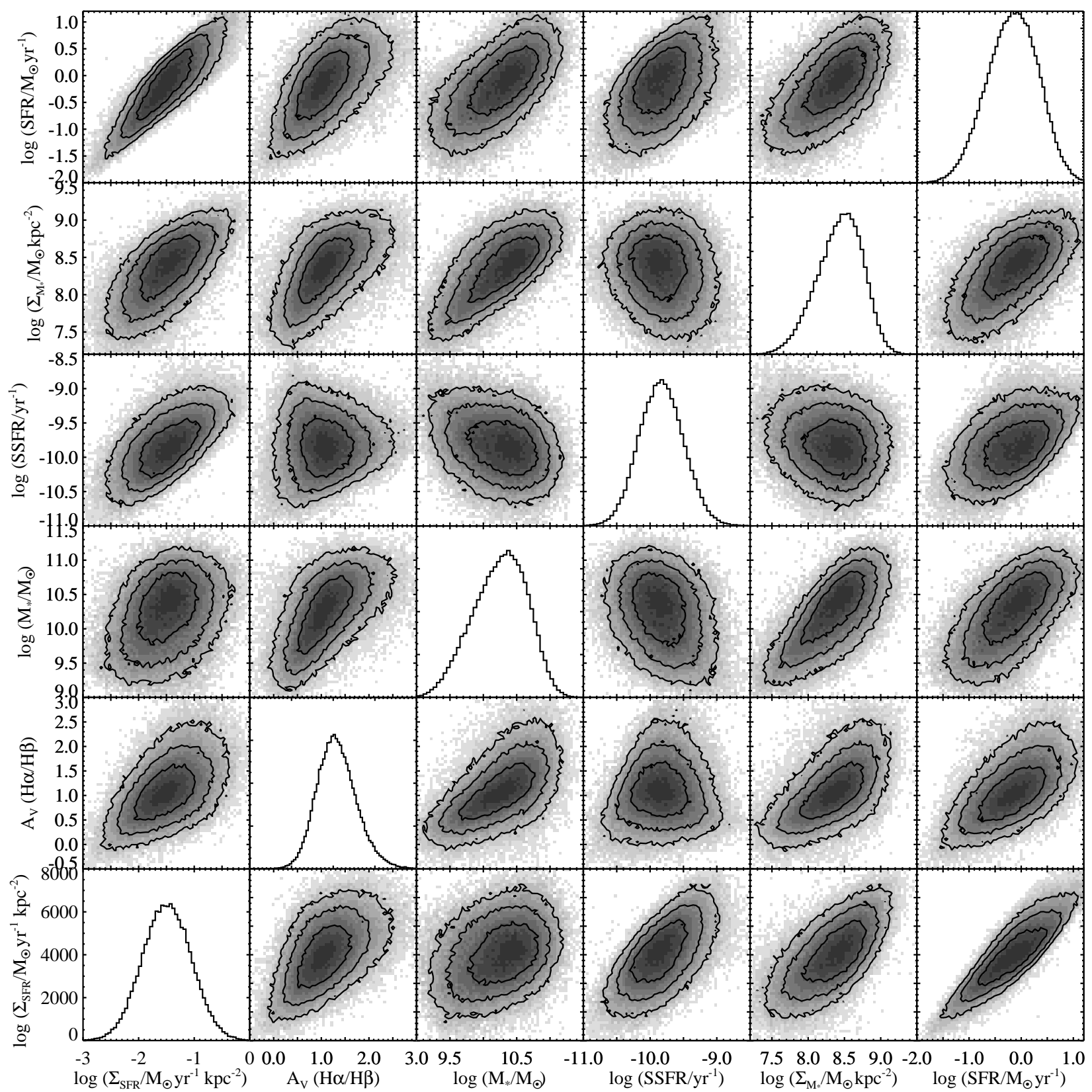

FIG. 1.- Correlations among the physical parameters of our Sample A galaxies. For panels where the $x$ - and $y$-axis are the same, histograms are shown. Note that all quantities except $M_{*}$ are measured within the SDSS fiber aperture.

In the previous section we found that the $\mathrm{Na} D$ residual increases with $A_{V}$. In this section, we study how the $\mathrm{Na} D$ residual evolves with the other galaxy parameters that we are interested in: SFR, $\Sigma_{\mathrm{SFR}}, M_{*}, \Sigma_{M_{*}}$, SSFR. We define galaxies with a Na D residual $>0.8 \AA$ as strong ISM Na D absorption galaxies. This definition is somewhat arbitrary, however, our conclusions are not very sensitive to the threshold we adopt.

Deep images of the edge-on starburst galaxy M82 in the Xray, $\mathrm{H} \alpha$, and $8 \mu \mathrm{m}$-bands (Ohvama et al. 2002; Mutchler et al. 2007; Engelbracht et al. 2006) indicate that gas and dust are being driven out along the galaxy minor axis in a weaklycollimated bipolar outflow (See Figure 3). Imaging of the $\mathrm{H} \alpha$ and soft X-ray emission shows that this wind geometry is common in starburst galaxies (e.g., Lehnert \& Heckman 1996; Strickland et al. 2004). Thus, absorption line studies of nearly face-on systems (low $i$ ) should probe gas in the outflow, while observations of nearly edge-on systems (high $i$ ) will not; they may, however, be sensitive to gas in the disk. In keeping with this picture, Heckman et al. (2000) found that there is a high probability $(\sim 70 \%)$ of detecting outflowing gas in absorption in starburst galaxies with inclinations less than $60^{\circ}$. Based on these results, we split our Sample A into two sub-samples with $i<60^{\circ}$ and $i>60^{\circ}$.

Figure 4 shows how the fraction of galaxies with $\mathrm{Na} \mathrm{D}$ residuals greater than $0.8 \AA$ changes with various galaxy parameters for our two samples split by inclination. The frac- 

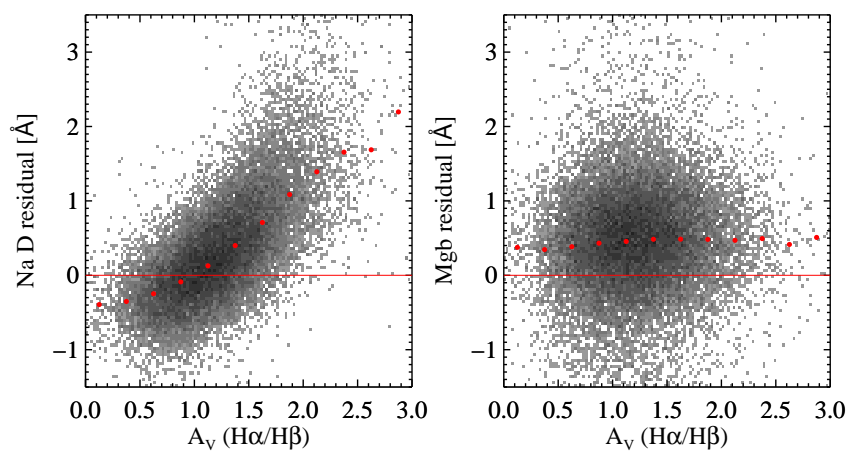

FIG. 2.- Residual Na D (left) and Mg $b$ (right) absorption versus dust attenuation for Sample A galaxies with S/N $>15$. Red dots indicate the median. The residual absorption is measured after subtraction of the best fit stellar continuum model. Dust attenuation is measured from the $\mathrm{H} \alpha$ and $\mathrm{H} \beta$ nebular lines. The strong positive correlation in the left panel is evidence that the excess $\mathrm{Na} \mathrm{D}$ absorption arises in the dusty ISM. The lack of correlation for $\mathrm{Mg} b$ is expected since this is an excited photospheric feature that does not have a counterpart in the ISM.

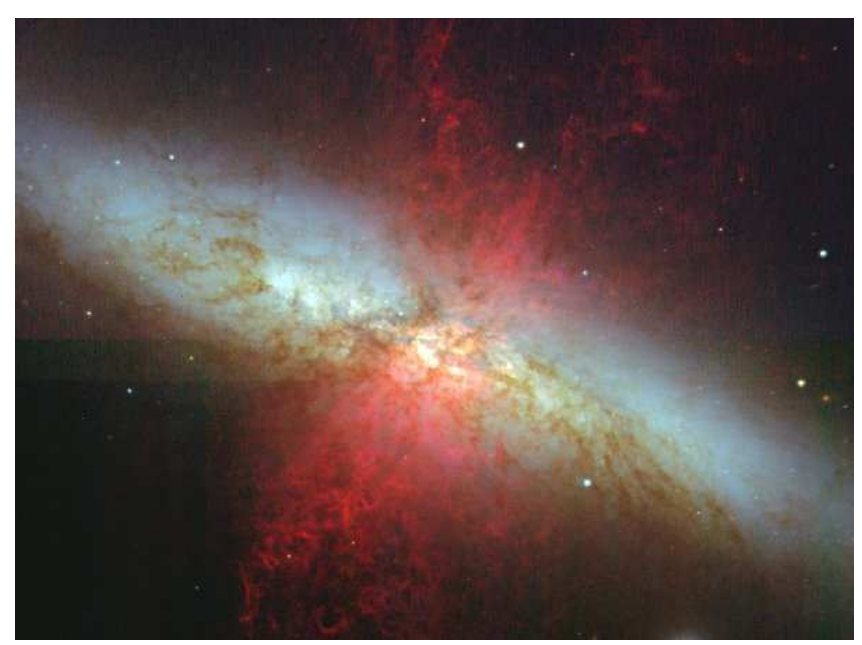

FIG. 3.- The galactic wind in the prototypical starburst galaxy M82. The color-composite image was constructed by the Hubble Heritage team from $\mathrm{B}, \mathrm{V}, \mathrm{I}$, and $\mathrm{H} \alpha$ images obtained with the Hubble Space Telescope Advanced Camera for Surveys (Mutchler et al. 2007). The galaxy has an inclination of $i \approx 80^{\circ}$. Outflowing gas is visible as a bi-polar cone of $\mathrm{H} \alpha$ emission (red) extending along the galaxy minor axis. If this wind geometry is common in disk galaxies, then we would expect to observe blueshifted $\mathrm{Na} \mathrm{D}$ when our sightline probes the wind bi-cone at $i<60^{\circ}$. The dusty filaments evident in the disk may give rise to $\mathrm{Na} \mathrm{D}$ absorption at the galaxy systemic velocity.

tion increases rapidly with all of the galaxy properties, except SSFR, for both low (black) and high (red) inclination samples. The highly inclined galaxies have stronger $\mathrm{Na} \mathrm{D}$ residuals; in $\$ 4.1$ we will argue that this strong $\mathrm{Na} \mathrm{D}$ absorption arises in the disk. Galaxies with strong ISM Na D absorption make up only a small part of the full sample; however, they dominate the populations with strong star formation, high stellar mass, and high dust attenuation. The blue vertical line in each panel marks the place where the fraction of galaxies with $i<60^{\circ}$ and strong $\mathrm{Na} \mathrm{D}$ reaches $5 \%$ and begins to rise steeply.

\section{DATA ANALYSIS}

In this section, we describe how we measure the properties of the ISM Na D absorption. As discussed in $\$ 2.3$, the low $\mathrm{S} / \mathrm{N}$ of the individual spectra will lead to large uncertainties
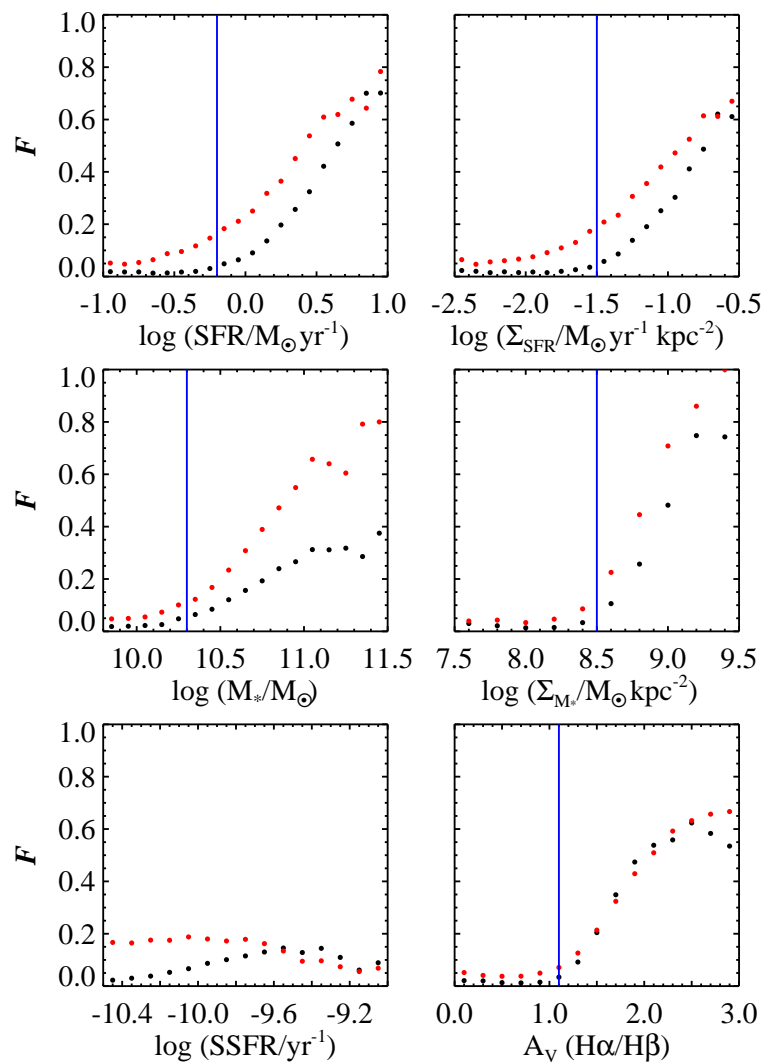

FIG. 4.- The fraction of strong Na D residual galaxies as a function of different galaxy parameters. The fraction is measured as $F=N(\mathrm{EW}>0.8) / N_{\text {bin }}$, where $N(\mathrm{EW}>0.8)$ is the number of galaxies with $\mathrm{Na} \mathrm{D}$ residual $>0.8 \AA$ in a given bin and $N_{\text {bin }}$ is the total number of galaxies in the bin. The black dots represent galaxies in Sample A with $i<60^{\circ}$ and red dots represent the galaxies with $i>60^{\circ}$. The blue vertical line in each panel marks the place where the fraction of $i<60^{\circ}$ galaxies with strong $\mathrm{Na} \mathrm{D}$ residual reaches $5 \%$ and begins to rise steeply.

in the measured $\mathrm{Na} \mathrm{D}$ EW. The median $\mathrm{Na} \mathrm{D}$ EW error for Sample $\mathrm{A}$ is $\sim 1.5 \AA$, which is greater than the strength of the interstellar absorption in most galaxies. By stacking (averaging) galaxy spectra we avoid this $\mathrm{S} / \mathrm{N}$ issue and are able to use samples that are relatively complete. The steps of our analysis are as follows:

- Stack spectra in different bins of galaxy physical parameters to create high $\mathrm{S} / \mathrm{N}$ composite spectra. The parameters used in our stacking analysis are galaxy inclination $(i), M_{*}, \Sigma_{M_{*}}, \mathrm{SFR}, \Sigma_{\mathrm{SFR}}, \mathrm{SSFR}$, and $\mathrm{A}_{\mathrm{V}}$.

- Fit the CB08 stellar population models to the stacked spectra generated in the first step. The aim is to separate stellar and ISM Na D absorption. Divide each spectrum by the best fitting continuum model.

- Fit the interstellar Na D line in each continuumnormalized spectrum. Return the EW, line centroid, line width, optical depth, and covering factor.

- Look for correlations between the properties of the gas and the galaxy physical properties.

Each of these steps is described in more detail below. 


\subsection{Stacking galaxy spectra}

The galaxy spectra are first corrected for foreground Galactic attenuation using the dust maps of Schlegel et al. (1998), transformed from vacuum wavelengths to air, and shifted to the restframe using the redshift determined by the SDSS specBS pipeline. We then normalize each spectrum to its median flux between 5450 to $5550 \AA$, where the spectrum is free of strong absorption and emission lines. The normalized restframe spectra in a given parameter bin are averaged ("stacked") using the following weighting scheme:

$$
f_{\text {comp }}(\lambda)=\frac{\sum_{i=1}^{n} \operatorname{mask}(i, \lambda) \times f(i, \lambda)}{\sum_{i=1}^{n} \operatorname{mask}(i, \lambda)}
$$

where the sum is over $i=1,2, \ldots, n$ galaxies with normalized flux $f(i, \lambda)$. We set $\operatorname{mask}(i, \lambda)=0$ for bad pixels (identified in the SDSS mask array), and $\operatorname{mask}(i, \lambda)=1$, otherwise. This method gives equal weight to all spectra, but excludes bad pixels from the composite.

We adopt an adaptive binning approach to produce composite spectra in different parameter bins with equal $\mathrm{S} / \mathrm{N}$. The galaxies are sorted by the physical parameter of interest and added to the stack one at time, from lowest to highest. After each addition, the $\mathrm{S} / \mathrm{N}$ of the continuum near $\mathrm{Na} \mathrm{D}$ is computed. The process is repeated until a $\mathrm{S} / \mathrm{N}$ of 300 is reached. The value of the physical parameter of interest in each bin is computed from the median of the individual measurements.

\subsection{Fitting the Continuum}

The aim of this study is to use the ISM Na D absorption to probe galactic winds in star forming galaxies. However, as discussed in $\$ 2.3$, we can not disregard the fact that interstellar $\mathrm{Na} \mathrm{D}$ absorption is superimposed on stellar Na D absorption. In previous studies of LIRGs and ULIRGs (Martin 2005, 2006; Rupke et al. 2005a. b), stellar Na D absorption was estimated from $\mathrm{Mg} \mathrm{I}$, and deemed to contribute only a small fraction $(<10 \%)$ to the total line EW. However, in the investigation of 32 far-IR-bright starburst galaxies, Heckman et al. (2000) found that $>40 \%$ of the galaxies (mostly edge-on systems) had strong stellar contamination. It is well-known that $\mathrm{Na} \mathrm{D}$ is strong in the spectra of cool stars, with a peak strength in the range from K3 to M0 (Jacoby et al. 1984). These stellar types are expected to make a significant contribution to the current sample since the SDSS spectra are obtained through a $3^{\prime \prime}$ circular fiber aperture that samples the central 3-12 kpc of our current sample. In these regions, bulge K-giants are likely to make a strong contribution to the integrated the stellar light (Heckman 1980; Bica et al. 1991).

To separate the stellar and ISM contribution to the Na D absorption we model the stellar continuum of each stacked spectrum using the CB08 stellar population synthesis models. In the Appendix, we show that our results are insensitive to the choice of stellar population models. Our continuum fitting procedure is nearly the same as that used in fitting individual spectra (see \$2.1). The main difference between the individual and stacked spectra is that the stacked spectra have much higher S/N. This makes it possible for us to constrain the stellar contribution using a larger number of SSP templates. We therefore fit the stacked spectra with all 40 templates (4 metallicities and 10 ages) simultaneously. This allows the best-fit model to consist of a mixture of metallicities, unlike the situation for the individual spectra $(\$ 2.1)$.

The He I $\lambda 5876$ nebular emission line on the blue side of $\mathrm{Na}$ I $\lambda 5890$ is broad enough to slightly influence our mea- surement of Na D. Since the line presumably originates in $\mathrm{H}$ II regions behind the $\mathrm{Na} \mathrm{D}$ absorbing gas, we include it in our continuum model. We fit a Gaussian to the blue wing of He I emission and add a model of the full line to the stellar continuum before normalizing the spectrum.

Observationally, in local galaxies, galactic winds are a general consequence of high SFR or $\Sigma_{\text {SFR }}$ (Lehnert \& Heckman 1996); they are common in galaxies above a $\Sigma_{\mathrm{SFR}}$ threshold of $0.1 M_{\odot} \mathrm{yr}^{-1} \mathrm{kpc}^{-1}$ (Heckman 2002). To illustrate our continuum and absorption line fitting procedure, we randomly select 2000 galaxies with $\Sigma_{\mathrm{SFR}}>0.1 M_{\odot} \mathrm{yr}^{-1} \mathrm{kpc}^{-2}$ from Sample $A$ and stack them. Figure 5 shows the resulting stacked spectrum (black line) and our stellar continuum fit (red line). We highlight the regions near Mg I (left insert drawing) and $\mathrm{Na}$ I (right insert). The vertical black lines mark the restframe wavelength center of the $\mathrm{Mg}$ I $\lambda \lambda 5167,5173,5184$ triplet, [N I] $\lambda 5198$, He I $\lambda 5876$, and the Na I $\lambda \lambda 5890,5896$ doublet. A Gaussian fit to the He I emission line is shown in cyan. From this plot, we infer that: (1) the stellar continuum model provides a very good fit overall; (2) the stellar contribution of the $\mathrm{Na} \mathrm{D}$ absorption is as high as $\sim 80 \%$ on average. Since the full Sample A includes galaxies with lower $\Sigma_{\text {SFR }}$ and a reduced incidence of strong ISM Na D absorption, we expect the stellar contribution to the $\mathrm{Na} \mathrm{D}$ line to be even larger in some cases.

\subsection{Measuring Interstellar Na D Absorption Line Profiles}

There are two common methods of measuring absorption line profile parameters: (1) fitting simple functional forms (such as Gaussian or Voigt profiles) to the intensity as a function of wavelength; (2) making more complex models, in which the intensity profiles are direct functions of physical parameters (velocity, optical depth, and covering fraction; e.g., Rupke et al. 2005a). The first solution is mainly based on mathematics rather than physics, and it is widely used for single lines or unblended doublets or multiplets. The study of unblended transitions is much easier than blended ones. For the blended doublet or multiplet, we prefer the second method since on one hand, the profile shape is readily understood in terms of a set of physical parameters, and on the other hand, different model assumptions can be tried. For the blended Na doublet, we choose to use the second method. We briefly summarize Rupke's model below and refer the reader to Rupke et al. (2005a) for more details.

Previous studies of $\mathrm{Na} \mathrm{D}$ absorption troughs in ULIRGs strongly suggest the assumption of complete continuum coverage is not met (Martin 2005, 2006; Rupke et al. 2005a.b). These studies also found super-thermal $\mathrm{Na} \mathrm{D}$ line widths ( $b \sim 300 \mathrm{~km} \mathrm{~s}^{-1}$ ), which can arise from multiple fragmented shells of cool gas traveling at different velocities (Fujita et al. 2009). When stacking many hundreds of galaxy spectra, absorption lines with different velocities, line widths, optical depths and covering factors will be blended. Jenkins (1986) explored this situation extensively using monte-carlo simulations, and determined that reasonable results can be obtained for the ensemble properties of the absorbers provided the distribution function of their individual properties is not too unusual. Results from stacked spectra may, in fact, be more robust than those from individual galaxies, because the number of blended components is larger.

We adopt the partial covering model for the current study and assume a covering factor, $C_{f}$, which is independent of velocity. In this case, the general expression for the intensity 


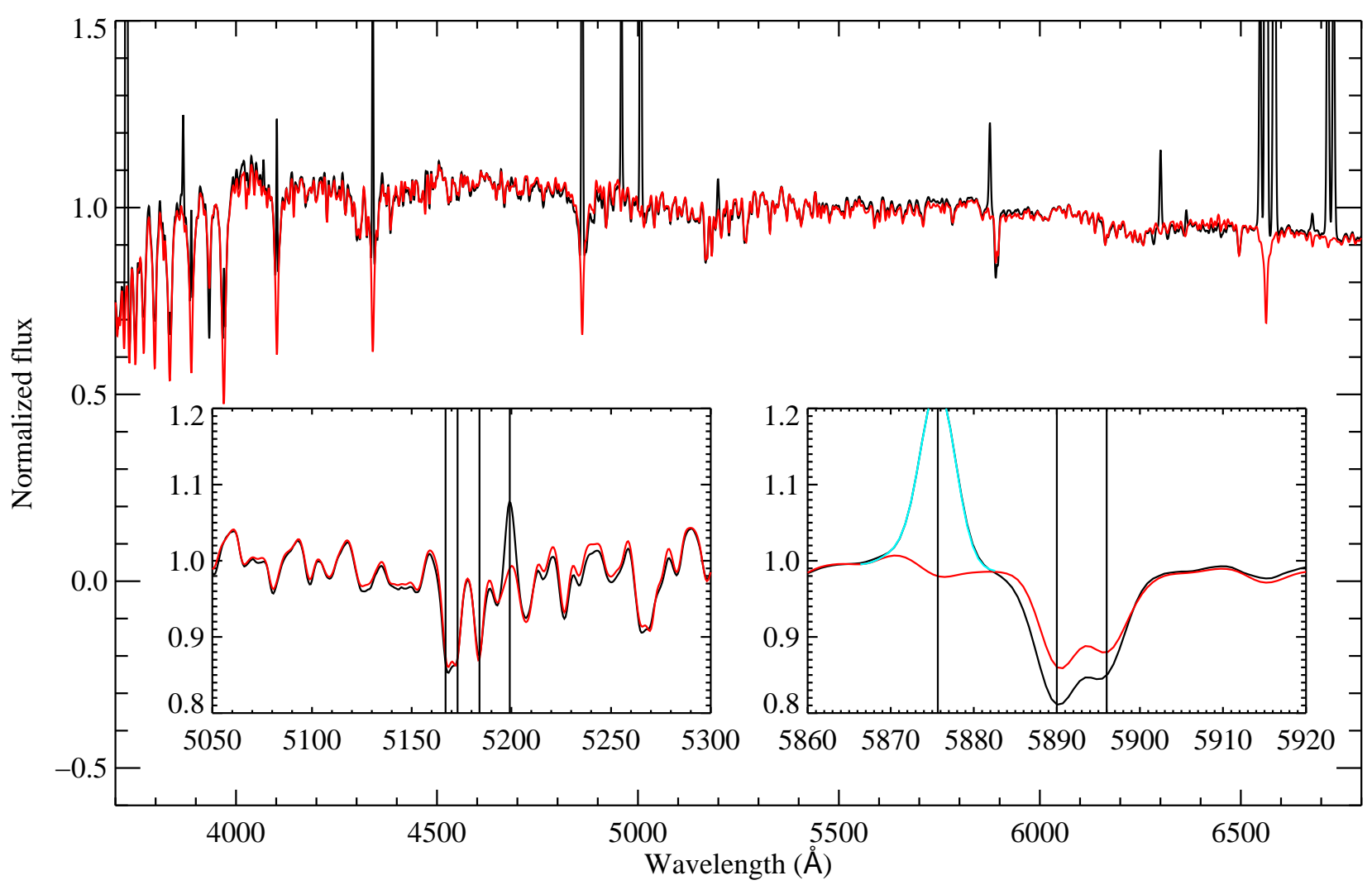

FIG. 5.- An example of a stacked spectrum (black) and its best-fit continuum model (red). The spectrum is a composite of 2000 randomly selected galaxies in Sample A with $\Sigma_{\mathrm{SFR}}>0.1 M_{\odot} \mathrm{yr}^{-1} \mathrm{kpc}^{-2}$. The two insert drawings highlight regions near the $\mathrm{Mg}$ I (left) and Na I lines (right). The vertical black lines mark the restframe wavelength of the Mg I $\lambda \lambda 5167,5173,5184$ triplet, the [N I] $\lambda 5198$, the He I $\lambda 5876$ emission line, and the Na I $\lambda \lambda 5890,5896$ doublet. A Gaussian fit to the He I emission line is shown in cyan.

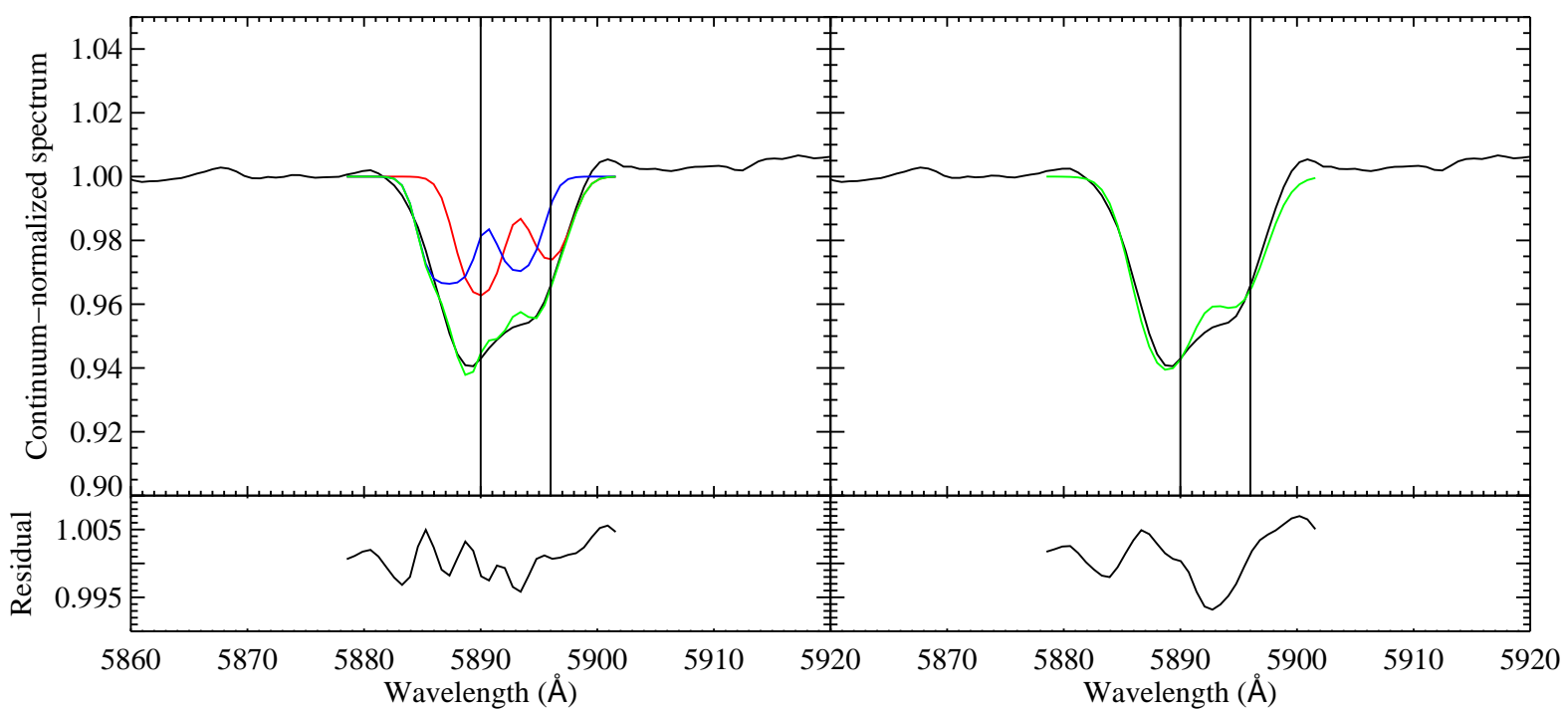

FIG. 6.- An example of our Na D line profile fits. The back line in the top panel shows the spectrum from Figure 5 normalized by its best fit continuum model. The left panel illustrates our two-component Na D fit: the red line represents the systemic component; the blue line is the outflow (blue-shifted) component; and the green line shows the combined fit. For comparison, we also show a single component fit in the right panel. The residuals of are shown in the bottom panels. The two component fit does a better job of modeling the shape of the line profile. 
of a doublet, $I(\lambda)$, assuming a continuum level of unity, is

$$
I(\lambda)=1-C_{f}+C_{f} e^{-\tau_{B}(\lambda)-\tau_{R}(\lambda)} .
$$

where $\tau_{B}(\lambda)$ and $\tau_{R}(\lambda)$ are the optical depth of the blue and red components of the doublet, respectively. For $\mathrm{Na} D, \tau_{B} / \tau_{R}=2$. Under the curve-of-growth assumption, the optical depth $\tau$ of a line can be expressed as:

$$
\tau(\lambda)=\tau_{0} e^{-\left(\lambda-\lambda_{0}\right)^{2} /\left(\lambda_{0} b / c\right)^{2}}
$$

where $\tau_{0}$ and $\lambda_{0}$ are the central optical depth and central wavelength of the line and $b$ is the line width. The four physical parameters of this model are:

- Outflow velocity $\left(v_{\text {off }}\right)$, which measures the shift of line profile centroid relative to the galaxy's systemic velocity $\left(v_{\text {sys }}\right)$.

- Optical depth at line center $\left(\tau_{0}\right)$ of the absorbing clouds, which influences the depth and intensity ratio of the two lines of the doublet.

- Covering factor $\left(C_{f}\right)$ of the absorbing clouds, which determines the residual flux at line center.

- The Doppler width $(b)$ of the absorption lines.

Given the low resolution of our spectra $\left(\sim 150 \mathrm{~km} \mathrm{~s}^{-1}\right.$ FWHM) and the blended nature of the profiles, there are some degeneracies between optical depth and covering factor which both influence the depth of the lines. The line width and the outflow velocity are more robust to degeneracies.

In the top panel of Figure 6 we show the stacked spectra of Figure 5 after continuum normalization. The rest wavelengths of the two sodium lines are marked by vertical lines. The line profiles appear asymmetric, with some of the gas blue-shifted relative to the systemic velocity of the galaxy. We hypothesize that there is an absorption component at the systemic velocity that arises in the disk, and an outflow (blue-shifted) absorption component that arises in a galactic wind. Systemic and outflow absorption line components were identified in previous studies of individual starbursts (c.f., Martin 2005), and in composite spectra of intermediate redshift galaxies (Weiner et al. 2009). We consider the origin of the two line components further in \$4.1 where we examine the inclination dependence of the $\mathrm{Na} \mathrm{D}$ line profile.

We fit the $\mathrm{Na} \mathrm{D}$ line profile with two absorption components, as shown in Figure 6, with the velocity of one component fixed at $v_{\text {sys }}$ (red). The blue line in Figure 6 denotes the outflow component. We also show the single component fit in the top-right panel (green). Comparing the residuals of these two fits, we find that the two component fit is superior. To aid in breaking the degeneracy of the two components, we have also fixed the line width of the systemic component to the width of the He I emission line. We assume that the He I line profile reflects the kinematics of $\mathrm{H}$ II regions in the disk, and that the gas producing the systemic $\mathrm{Na} \mathrm{D}$ absorption shares these kinematics. To have an idea of how the final results depend on this assumption, we tried two other methods: (1) fixing the systemic component to 1.5 times the width of the He I emission line; and (2) letting it vary in the range of [11.5] He I width. While method (2) gives very similar fitting parameters as that of fixing the width to He I, method (1) produces larger spectral residuals and the fitted parameters show more scatter with galaxy physical properties. We therefore fix the linewidth of the systemic component to He I.
As noted in $\$ 2.3$, there are cases where the best-fit continuum model has stronger $\mathrm{Na} \mathrm{D}$ absorption than the actual data. The reasons for this are still not fully understood. One possibility is that the young stars used in the empirical stellar libraries of CB08 have some excess absorption at $\mathrm{Na} \mathrm{D}$ due to the Milky Way's ISM. However, a comparison with fully theoretical models (see Appendix) suggests that such absorption, if present, is not very strong. Another possibility is that a combination of 40 instantaneous burst models with a range in age and metallicity is insufficient to represent the the true galaxy star formation history. Degeneracies may also limit the ability of our fitting code to arrive at the optimal solution. We cannot rule out continuum fitting errors, however, we find this explanation less likely, given the high quality of the fits elsewhere in the spectrum (c.f., Fig. 5). The final possibility is that Na D is sometimes in emission. This is plausible because Na D is a resonance absorption transition. (Each absorbed photon is re-emitted isotropically.) Whether $\mathrm{Na} \mathrm{D}$ is detected in absorption or emission in a given spectrum depends on the relative geometry and velocity of the gas and stars probed by the fiber. We note that the emission appears slightly redshifted and it is seen predominantly in galaxies that are face-on and have low dust attenuation. This suggests that we are seeing through the disk to the back side of the expanding bi-polar outflow. As the disk becomes progressively more dusty or inclined the emission from the far side of the disk is attenuated. Emission was not detected in previous studies of LIRGs and ULIRGs due to the dusty nature of these sources. However, Phillips (1993) saw a clear Na D P-Cygni profile (blushifted absorption, redshifted emission) in the disk galaxy NGC 1808. In the DEEP2 Mg II study, Weiner et al. (2009) found Mg II emission in a subset of the galaxies. The emission tends to be in the bluest of the galaxies. In some of them it is visible in the individual spectra, not just the stack. They don't have inclination information for the high $-z$ galaxies, but it is natural to think that bluer galaxies are lower inclination on average.

We include the objects with emission $\mathrm{Na} \mathrm{D}$ residuals in the stacking and accommodate the possibility of $\mathrm{Na} \mathrm{D}$ emission by allowing the systematic component in our line profile fit to be in either absorption or emission. We note that in some cases a substantial systemic emission component is fit, even when the line profile appears to be completely in absorption (c.f., the first panel of Figure 8). In such cases, large residuals result from imposing the requirement that the systemic component be in absorption (note the strong asymmetry of the line profile). We believe these fits are robust and represent real cases of superimposed $\mathrm{Na} \mathrm{D}$ emission from the disk and absorption from the outflow. Figure 9 shows very smooth trends with galaxy physical properties as the systemic component transitions from absorption to emission, suggesting that our code generally does a good job of distinguishing the outflow and systemic components whether the latter is in absorption or emission. We have therefore chosen to allow the systemic component to be in emission; for simplicity, we implement this by allowing for negative covering factors.

\section{PROBING GALACTIC WINDS USING STACKED SPECTRA}

The dependence of the outflow properties on galaxy physical properties is of great interest because it can help clarify the influence of SFR and stellar mass on the wind kinematics. We can also learn which galaxies host outflows and thereby gain insight into the origin of metals in the IGM. These empirical results can, in turn, be incorporated into numerical simula- 


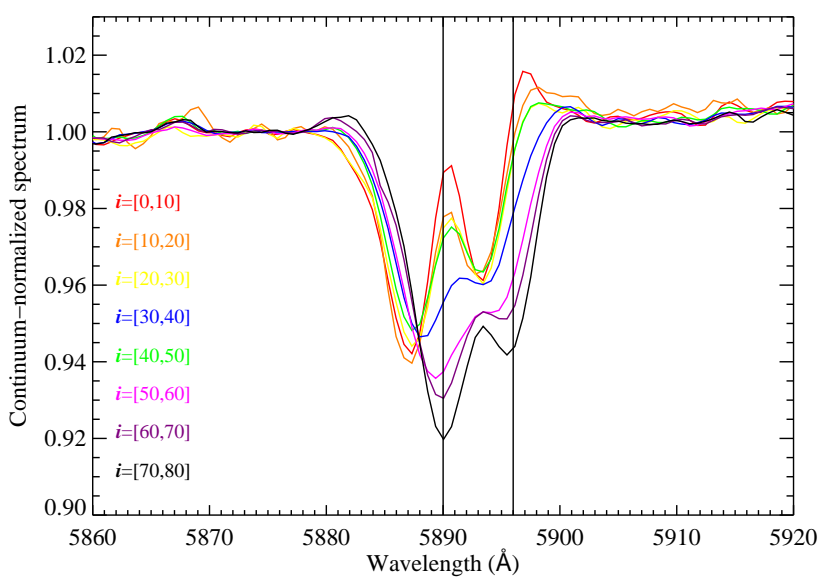

FIG. 7.- Continuum-normalized spectra binned by galaxy inclination. Different inclination bins are indicated by different colors, with the galaxies closest to face-on in red and the galaxies closest to edge-on in black.

tions of galaxy formation and evolution.

The data analysis method described in $\S 3$ is applied into our star forming galaxy sample in this section. We explore the effect of changes in galaxy inclination in \$4.1, and examine how the outflow and systemic components of the ISM $\mathrm{Na} \mathrm{D}$ absorption evolve with various galaxy physical properties in \$4.2. This is the first time outflow properties have been studied with large complete sample in the local universe that includes normal star forming galaxies as well as starbursts.

\subsection{Trends with Galaxy Inclination}

It has been suggested that the outflow component of the $\mathrm{Na} \mathrm{D}$ absorption arises from ambient interstellar material that has been entrained and accelerated along the minor axis of the galaxy by a hot starburst-driven superwind (Heckman et al. 2000), while the systemic component of the absorption arises in the disk. Based on this simple picture, we expect the two $\mathrm{Na} \mathrm{D}$ components to have strong - and opposite - variations with galaxy inclination. We test our assumptions about the absorber geometry using spectra stacked in bins of galaxy inclination.

Galactic winds are ubiquitous in galaxies with $\Sigma_{\mathrm{SFR}}>$ $0.1 M_{\odot} \mathrm{yr}^{-1} \mathrm{kpc}^{-1}$. To test our picture of the wind outflow geometry, we therefore apply this $\Sigma_{\mathrm{SFR}}$ cut to our 'Sample A' galaxies, to select sources likely to host outflows. In Figure 7 we compare the interstellar $\mathrm{Na} \mathrm{D}$ absorption profiles for these galaxies in 8 different inclination bins. Three features are immediately obvious. First, the line profile depth at the rest wavelength of the doublet increases dramatically as inclination increases and galaxies are viewed more nearly edge-on. Second, the line center shifts to bluer wavelengths as inclination decreases and galaxies are viewed more nearly face-on. Third, the lines are not saturated — in which case the doublet ratio would be closer to 1:1 - nor are the profiles black at the line center, which would indicate complete covering of the source by the absorber.

Figure 8 illustrates our two-component absorption line fits to composite spectra in different inclination bins. The red lines show the component fixed at $v_{\text {sys }}$, the blue lines show the outflow component, and the green lines show the combined fit. The systemic component (red) evolves markedly from emission at low inclinations to become the dominant $a b-$ sorption component at high inclinations. The outflow component (blue) evolves strongly in the opposite sense, dominating the line profile in face-on systems, but becoming a minor contributor to the absorption in edge-on galaxies.

The equivalent width $(\mathrm{EW})$, velocity $\left(v_{\text {off }}\right)$, line width $(b)$, covering factor $\left(C_{f}\right)$ and optical depth at line center $\left(\tau_{0}\right)$ of the two absorption line components are shown as a function of inclination angle in Figure 9 . At $i<60^{\circ}$, the outflow velocity depends only weakly on inclination, and has a median value of $\sim 140 \mathrm{~km} \mathrm{~s}^{-1}$. Above $i=60^{\circ}$, the velocity drops precipitously. The line width of the outflow component decreases by $\sim 40 \%$ as galaxies go from face-on to edge-on, but shows considerable scatter. The EW of the outflow component changes smoothly and nearly linearly from $E W=0.7 \AA$ to at $i \sim 0^{\circ}$ to nearly 0.1 at $i=90^{\circ}$. This trend appears to be driven primarily by a decrease in the gas covering factor. The optical depth of the outflow component increases sharply above $i=60^{\circ}$. This may indicate that the wide-angle outflow of dusty material has a more transparent and fast-moving "core" $\left(i<60^{\circ}\right)$ and a surrounding sheath of optically-thick slower-moving entrained material $\left(i>60^{\circ}\right)$ - sort of a "turbulent boundary layer". However, the outflow component makes a relatively small contribution to the total $\mathrm{EW}$ in this regime, so some caution in interpreting the result is advised.

The systemic absorption component changes with inclination in the opposite sense of the outflow component: the line width increases with inclination by $\sim 20 \%$ and the EW increases, from emission at $i \sim 0^{\circ}$ to $0.7 \AA$ of absorption at $i \sim 90^{\circ}$. The lack of zero-velocity ISM absorption component at low inclinations is consistent with the result of Weiner et al. (2009), in which they found that in the bluest subset, which are likely to be galaxies viewed face-on, there was no component of absorption at systemic velocity. Again, most of this change can be attributed to the covering factor; the optical depth shows little trend with inclination, and considerable scatter. The scatter in Figure 9 represents a lower bound to the uncertainities in the fits.

The trends shown in Figure 9 are in agreement with our basic hypothesis that $\mathrm{Na} \mathrm{D}$ absorption arises in both the galaxy disk and in an outflow directed along the galaxy minor axis. Notably, the strength of the systemic component increases as galaxies are viewed more nearly edge-on and a greater path length through the disk is probed. This leads us to conclude that the systemic component arises in the warm neutral medium of the disk. These disk clouds could either be in the central star forming region, in which case they must be too massive or too pressure-confined to be affected by feedback from young star clusters, or the clouds could be in the outer more quiescent part of the disk which is probed by the fiber at high galaxy inclinations.

The outflow absorption component covers a wide angle, but seems stronger within $\sim 60^{\circ}$ of the disk rotation axis; it likely arises from a large-scale galactic wind, like that seen in Figure 3 Notably, the velocity is roughly constant at $i<60^{\circ}$, as expected if the outflow is radially directed. At $i=60^{\circ}$, the EW of the disk-like and outflow components are roughly equal. This is consistent with our understanding of the outflow and disk geometry: at $i<60^{\circ}$, the ISM Na D absorption is dominated by outflow, while the disk-like component becomes important at $i>60^{\circ}$. Based on these results, we will split "Sample A" into two sub-samples, "Sample B", with $i>60^{\circ}$, and "Sample C" with $i<60^{\circ}$. There are 60,211 and 80,414 galaxies in each sample, respectively. 


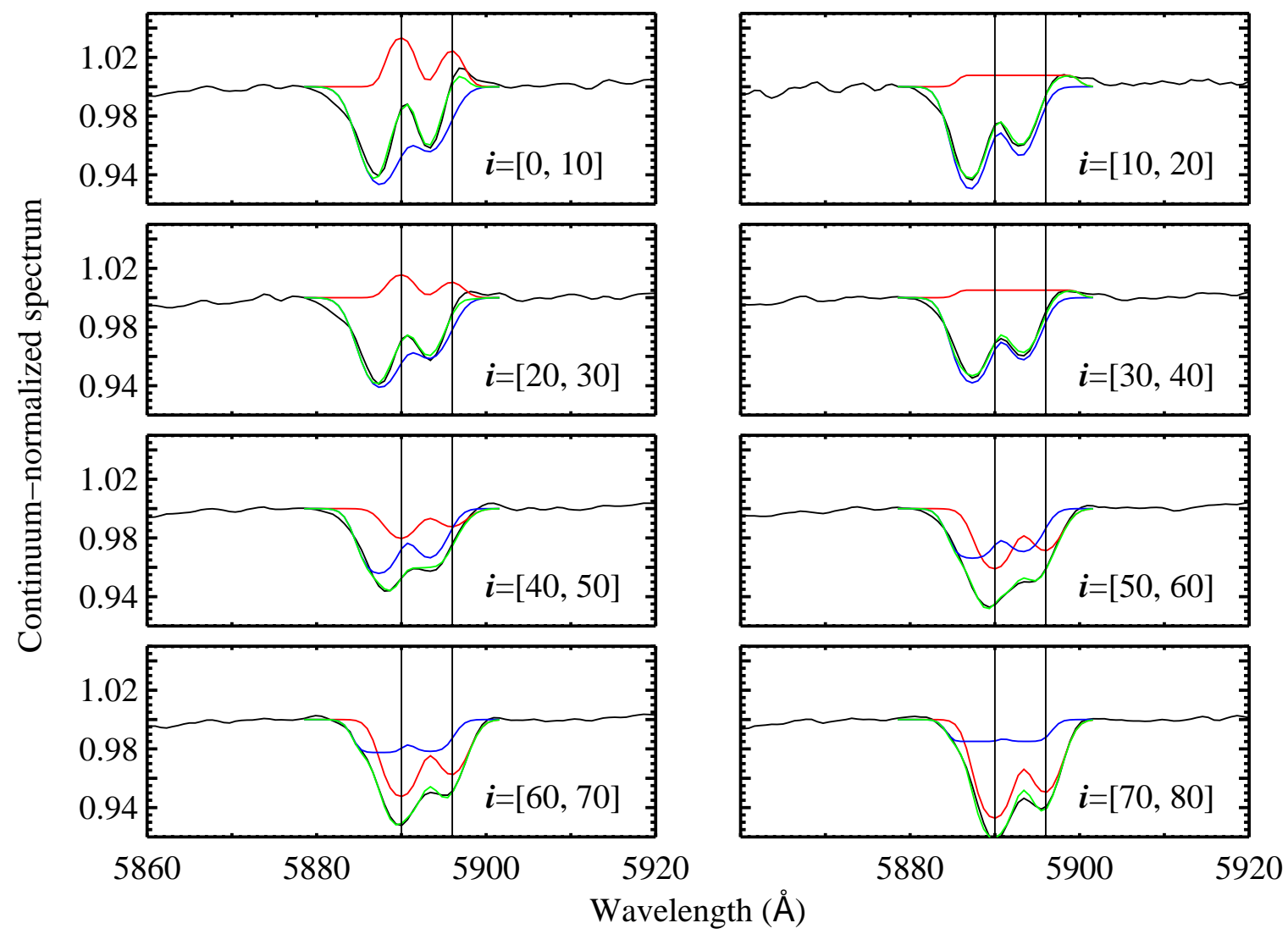

FIG. 8.- Absorption line profile fits to composite spectra in different inclination bins. The black lines show the continuum normalized composite spectra; the red line shows the absorption line component fixed to the galaxy systemic velocity; the blue line shows the outflow component; and the green line shows the combined fit. Note the appearance of Na D emission at the systemic velocity in the low inclination bins.

\subsection{Trends with Galaxy Physical Properties}

In this section we examine how the properties of the two $\mathrm{Na} \mathrm{D}$ absorption components (as derived from our constrained two-component fit) vary as a function of galaxy physical properties. "Sample B" and "Sample C" are used to study the disklike and outflow components, respectively. Our reasoning is that the absorbers in the disk are best studied in galaxies that are more nearly edge-on, while absorbers in the outflow are best studied in galaxies that are more nearly face-on.

We consider each galaxy physical parameter independently and use the adaptive binning approach described in $\$ 3.1$ to construct stacked spectra. We do not consider the full parameter range, but rather we define a lower boundary based where the fraction of strong $\mathrm{Na} \mathrm{D}$ absorbers (EQ $>0.8 \AA$ ) begins to rise steeply (see the blue lines in Fig. 4). The continuumnormalized spectra for eight different $\Sigma_{\mathrm{SFR}}$ bins from "Sample $\mathrm{C}^{\prime \prime}$ are shown in Figure 10 as an example. The strength of the absorption increases dramatically with increasing $\Sigma_{\text {SFR }}$. Figure 11 shows the two-component fits of these eight spectra.

We plot the parameters derived from our absorption line fits $\left(v_{\text {off }}, b, \mathrm{EW}, C_{f}, \tau_{0}\right)$ as a function of galaxy physical properties $\left(\Sigma_{\mathrm{SFR}}, \mathrm{A}_{\mathrm{V}}, \mathrm{SSFR}, M_{*}\right)$ for Samples B and C in Figures 12 and 13. We use these plots to help discern the galaxy physical parameters that have the greatest influence on the warm neutral medium in and outside of galaxy disks. However, the strong correlations among the galaxy physical parameters illustrated in Figure 1 make this exercise challenging. We also considered correlations with SFR and $\Sigma_{M_{*}}$ (both measured in the fiber aperature). Trends with these parameters are very similar to those found with $\Sigma_{\text {SFR }}$ and $M_{*}$, but typically weaker and/or nosier. For clarity we have not included SFR and $\Sigma_{M_{*}}$ in our subsequent analysis.

For the systematic component (Fig. 12), we note the following:

- Na D EW increases strongly and nearly linearly with $\log \left(\Sigma_{\mathrm{SFR}}\right), \log \left(M_{*}\right)$, and $\mathrm{A}_{\mathrm{V}}$ from negative values (emission) to $\sim 1.0 \AA$. We note that the line EW is sensitive to the velocity spread of the gas, the cloud covering factor and the optical depth.

- The line width (which is tied to the width of the He I line) also increases smoothly with all of the stacking parameters except SSFR. The strongest trend seen is a $60 \%$ increase in $b$ with stellar mass. Since Sample B galaxies are highly inclined, the line width probably reflects the rotation speed of the inner disk. The apparent correlation between $b$ and $\Sigma_{\mathrm{SFR}}, \mathrm{A}_{\mathrm{V}}$ most likely results from the correlation of $M_{*}$ with these parameters, as show in Figure 1

- The covering factor changes steeply, from negative val- 

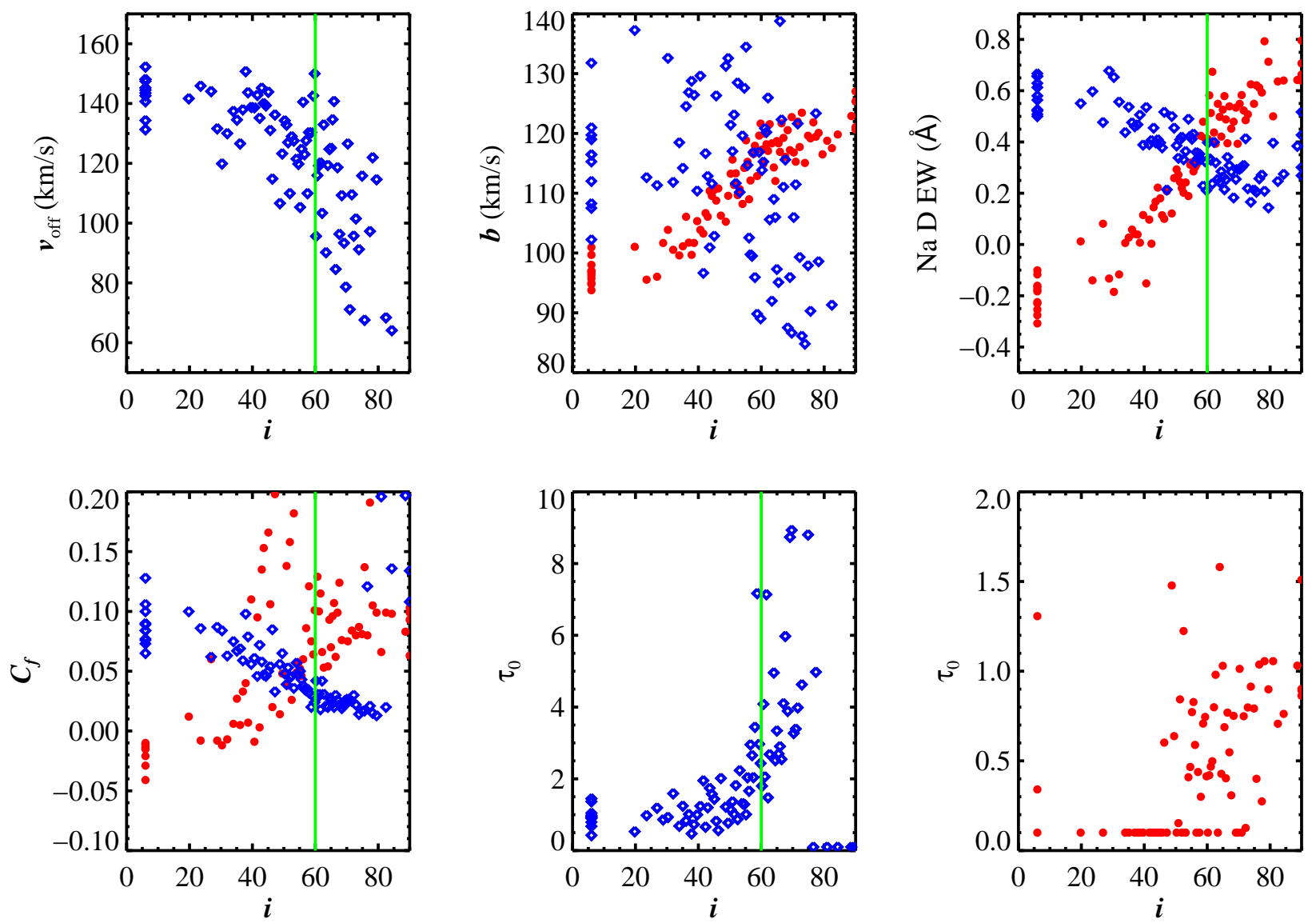

FIG. 9.- Derived parameters of the outflow and systemic Na D absorption components as a function of inclination angle. The blue diamonds represent the outflow component and the red circles represent the systemic component. The parameters shown include the velocity of the outflow component $\left(v_{\mathrm{off}}\right)$, the line width $(b)$, the covering factor $\left(C_{f}\right)$, the optical depth at line center $\left(\tau_{0}\right)$ and the line equivalent width $(\mathrm{EW})$.

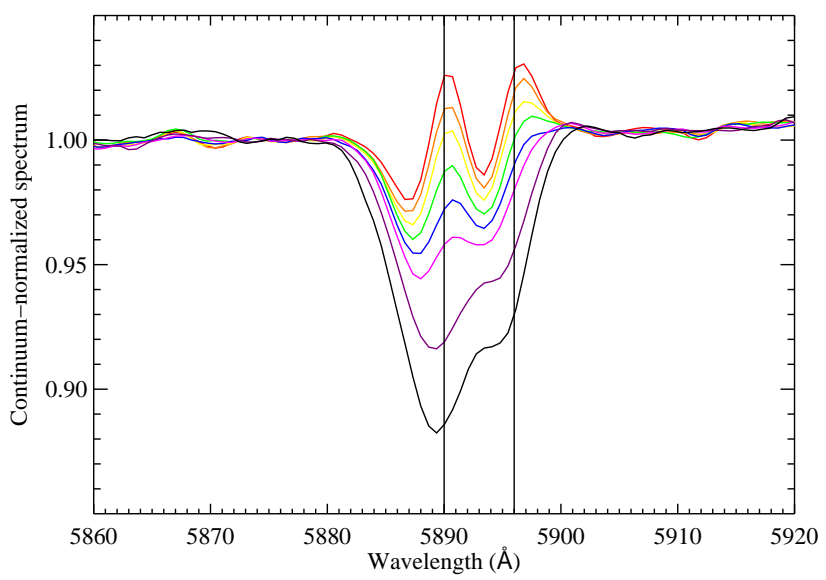

FIG. 10.- Continuum normalized composite spectra of galaxies in 8 different $\Sigma_{\mathrm{SFR}}$ bins drawn from Sample C $\left(i<60^{\circ}\right)$. As $\Sigma_{\mathrm{SFR}}$ increases, the absorption becomes stronger. $\Sigma_{\mathrm{SFR}}$ increases from red to purple.

ues (Na D emission) to $C_{f}=0.15$. The very small $\mathrm{Na} \mathrm{D}$ covering factor may be related to the low filling factor of neutral gas in galactic disks. The trends are similar to those identified with the EW, but noisier due degeneracies with $\tau_{0}$ exacerbated by the blended nature of our line profiles.

- The optical depth ranges from $\tau_{0}=0$ to $\sim 1.5$, and it does not depend strongly on any of the stacking parameters. In general, the optical depth is the least well constrained parameter that we measure.

Examining the changes in $b, C_{f}$, and $\tau_{0}$, we hypothesize that the changes in EW are driven primarily by the covering factor with an small additional contribution due changes in the line width. It is difficult to determine whether $\Sigma_{\mathrm{SFR}}, \mathrm{A}_{\mathrm{v}}$, or $\mathrm{M}_{*}$ are the primary driver of the trends. SSFR is clearly not an important parameter. We address the issue of correlations between the various physical parameters in $\$ 4.3$

In Figure 13 we explore the dependence of outflow Na D component on various galaxy properties. We note the following:

- The Na D EW increases with $\Sigma_{\mathrm{SFR}}, M_{*}$ and $\mathrm{A}_{\mathrm{V}}$ (similar to the disk component).

- The line width appears to scale strongly with $\Sigma_{\mathrm{SFR}}, M_{*}$ and $\mathrm{A}_{\mathrm{V}}$. The tightest correlation appears to be with $M_{*}$ : $b \propto M_{*}^{0.65}$.

- The covering factor is very low $\left(C_{f} \sim 0.04-0.12\right)$ and changes by a factor of three, at most. The strongest correlation is with $A_{V}$ and $M_{*}$. 


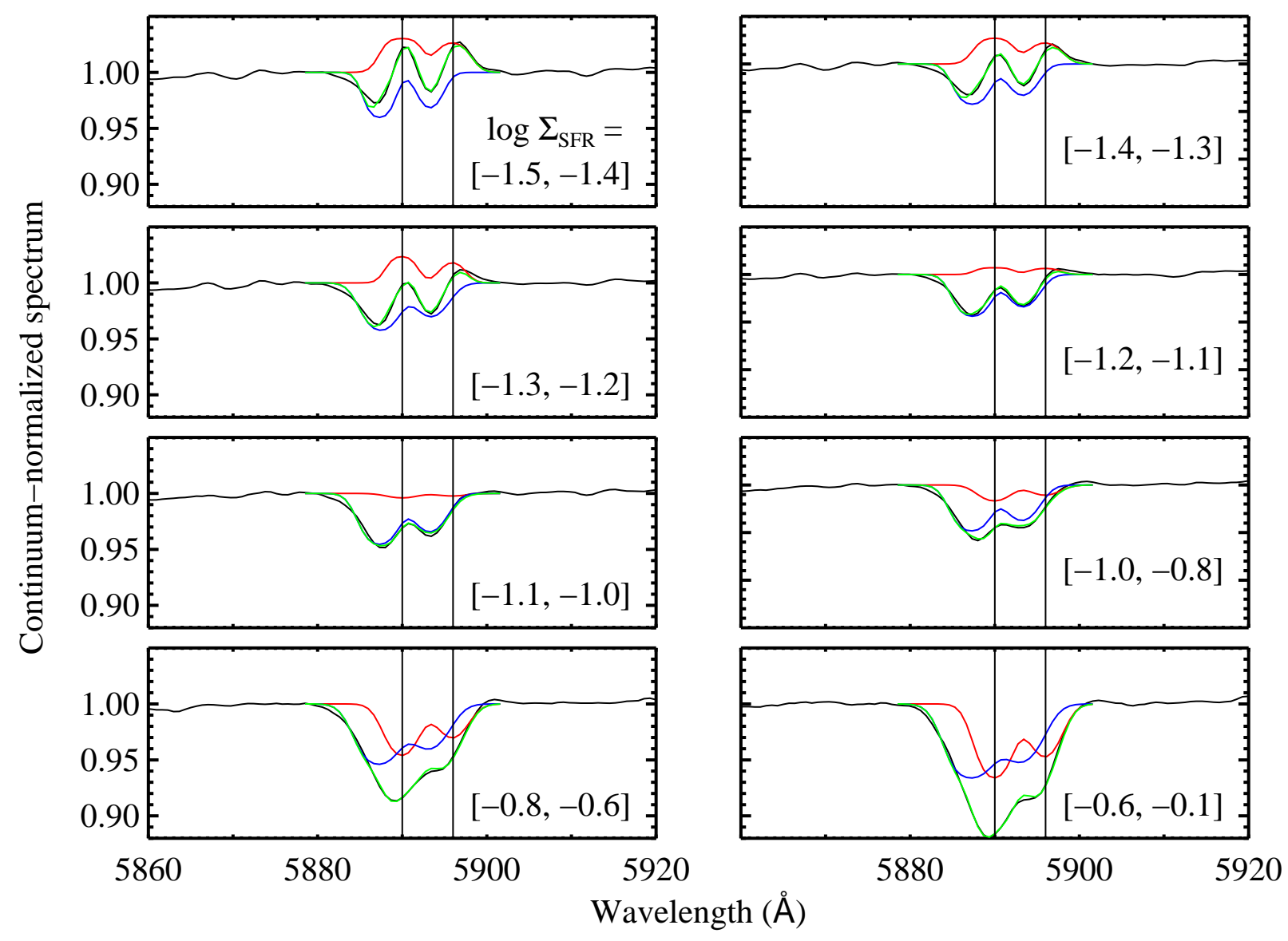

FIG. 11.- Absorption line profile fits to composite spectra in different $\Sigma_{\text {SFR }}$ bins. (The spectra are the same as those shown in Figure 10) The black lines show the continuum-normalized spectrum; the red line is the systemic (disk) absorption component; the blue line is the outflow component; and the green lines is the combined model. The range in $\log \Sigma_{\mathrm{SFR}}$ is given in the lower right of each panel.

- The lines range from unsaturated to mildly saturated $\left(\tau_{0} \sim 3\right)$ and $\tau_{0}$ displays a pronounced inverse correlation with $\mathrm{A}_{\mathrm{V}}$, and $\mathrm{M}_{*}$.

- The outflow velocity does not show any strong trend with galaxy physical properties. A very weak and noisy correlation is evident with $\Sigma_{\mathrm{SFR}}$ and possibly with $M_{*}$.

- No properties of the outflow component are well correlated with the specific star formation rate.

One possible interpretation of the observed trends in $C_{f}$ and $\tau_{0}$ is that in galaxies with low dust attenuation $\mathrm{Na} \mathrm{D}$ only survives in very dense clouds in the outflow. These clouds are optically thick and comparatively rare, so the average $\tau_{0}$ is high and the $\mathrm{Na} \mathrm{D}$ covering factor is very low. In galaxies with higher dust-to-gas ratios, $\mathrm{Na} \mathrm{D}$ is shielded from ionizing radiation in clouds with lower optical depths. Since these clouds are more numerous, the $\mathrm{Na} \mathrm{D}$ covering factor is higher and the average optical depth is lower. Thus, the mean optical depth decreases with $A_{\mathrm{V}}$ while the covering factor increases, as observed.

The lack of trends with SSFR is somewhat surprising since it might be expected that outflows depend on both the SFR and the potential well depth. One explanation is the strange shape of the SSFR vs. AV correlation (Fig 11). Galaxies with both low and high SSFR have low dust attenuation. This arises because low SSFR galaxies are predominately gas-poor massive galaxies with high dust-to-gas ratios, while high SSFR galaxies are predominantly gas-rich dwarfs with low metallicities and dust-to-gas ratios. At intermediate values of SSFR, galaxies display a wide range in dust attenuation. Clearly, dust shielding plays a key role in the detectablity of outflows using $\mathrm{Na}$ I.

The EW of the outflow component will depend on $b, C_{f}$, and $\tau_{0}$, although the influence of $\tau_{0}$ is minimal for galaxies with $\tau_{0}>1$. The EW depends linearly on $b$ and $C_{f}$, but the dynamic range probed by our sample is greater in $C_{f}$ (factor of $\sim 6$ vs. factor of $\sim 3$.) The EW thus primarily reflect changes in the covering factor. Since our measurement of the EW is less noisy than our measurement of $C_{f}$, we use the EW in examining tends with galaxy physical properties further below.

\subsection{Isolating the Primary Drivers of the Observed Correlations}

In figures 12 and 13 we identified $\mathrm{A}_{\mathrm{V}}, \Sigma_{\mathrm{SFR}}$, and $M_{*}$ as important physical parameters controlling the $\mathrm{Na} \mathrm{D}$ line profile. However, due to strong correlations among these parameters (Fig. 1), it is difficult to isolate the primary drivers of 

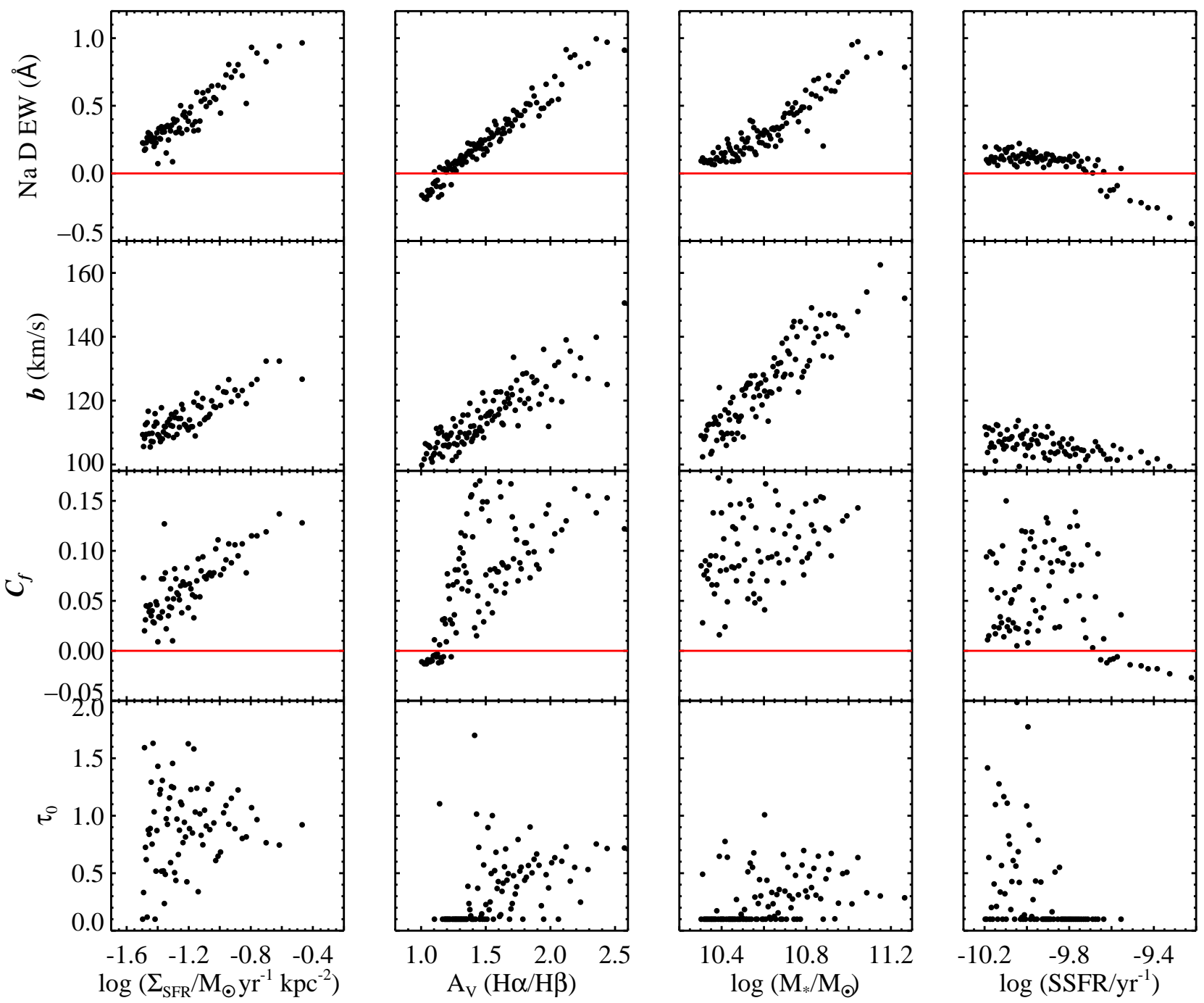

FIG. 12.- Derived parameters of the systemic Na D absorption component as a function of $\Sigma_{\mathrm{SFR}}, \mathrm{A}_{\mathrm{V}}, M_{*}, \mathrm{SSFR}$ for Sample B galaixes $\left(i>60^{\circ}\right)$.

the trends we observe. The correlations are sufficiently strong that it is not possible, for example, to look for trends with $\Sigma_{\mathrm{SFR}}$ at fixed $M_{*}$. A bin with a small range in stellar mass would also have a small range in $\Sigma_{\mathrm{SFR}}$. To isolate the most important physical parameters, we adopt the following approach. We take each bin in $M_{*}$ (for example) and further divide it into three equal sub-bins, sorting the galaxies by $\Sigma_{\text {SFR }}$. We are then able to examine trends between $\mathrm{Na} \mathrm{D}$ absorption and $M_{*}$ in low, medium, and high $\Sigma_{\mathrm{SFR}}$ bins (where the exact division between low, medium, and high changes with $M_{*}$ ). If $M_{*}$ is the primary driver of the trend, then no difference is expected between the sub-bins. Conversely, if $\Sigma_{\mathrm{SFR}}$ is the primary driver of the trend, then the three sub-bins will be strongly offset from one another at each value of $M_{*}$.

Figure 14 shows the Na D EW of the systemic component as a function of $M_{*}, \Sigma_{\mathrm{SFR}}$, and $\mathrm{A}_{\mathrm{V}}$ for galaxies in Sample B with $\log M_{*} / M_{\odot}>10.3$. In each panel the sample is split into three sub-bins according to the galaxy parameter labeled in the top-left corner. The black, red, and blue points indicate the low, medium, and high sub-bins respectively. The dominant driver of the observed trend (the parameter displaying the smallest offsets between the sub-bins) appears to be $A_{V}$. This suggests that high dust attenuation is important to keep Sodium in the disk neutral. A small residual correlation is evident with $\Sigma_{\mathrm{SFR}}$ and $\mathrm{M}_{*}$. A correlation between $\Sigma_{\mathrm{SFR}}$ and the $\mathrm{Na} \mathrm{D}$ covering factor is expected if $\Sigma_{\mathrm{SFR}}$ scales with the filling factor of molecular clouds, as suggested in Bigiel et al. (2008). The residual trend of EW with stellar mass is due to the larger velocity spread of the line in disk galaxies with larger rotation speeds.

Figure 15 shows the $\mathrm{Na} \mathrm{D} \mathrm{EW}$ of the outflow component as a function of $M_{*}, \Sigma_{\mathrm{SFR}}$, and $\mathrm{A}_{\mathrm{V}}$ for galaxies in Sample $\mathrm{C}$ with $\log \left(M_{*} / M_{\odot}\right)>10.3$. As in Figure 14, the galaxies are split into three sub-bins according to various physical properties. The plots with stellar mass on the $\mathrm{x}$-axis show very large discrepancies between high and low sub-bins in $\Sigma_{\mathrm{SFR}}$ and $\mathrm{A}_{\mathrm{V}}$, therefore, we infer that $M_{*}$ is the least important physical parameter in determining the EW. The most important parameter influencing the line EW appears to be $\Sigma_{\text {SFR }}$ followed closely by $A_{V}$. The physical explanation for these correlations is straightforward: star formation surface density determines the amount of material lofted above the disk and the dust attenu- 

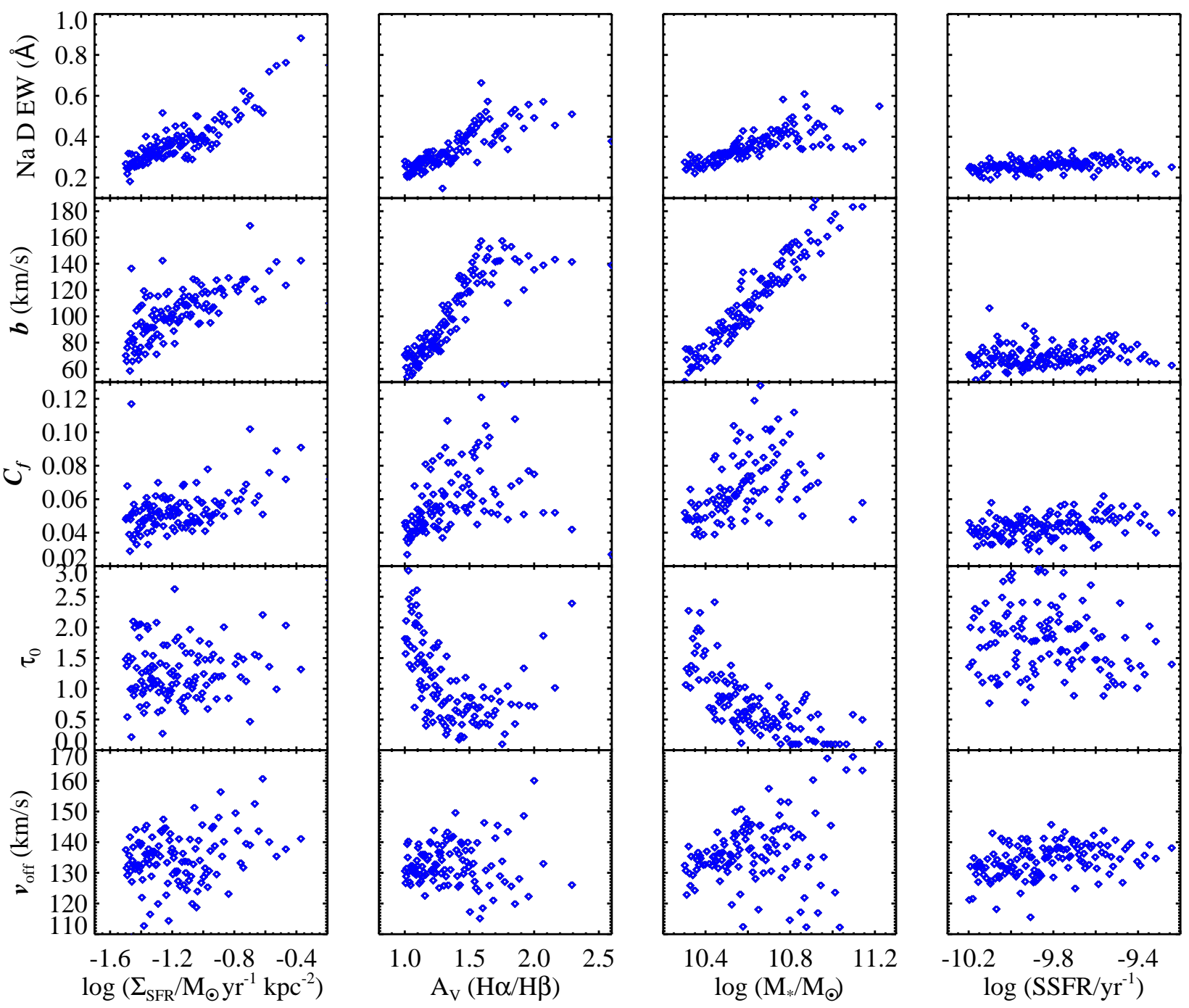

FIG. 13.- Derived parameters of the outflow component as a function of $\Sigma_{\mathrm{SFR}}, \mathrm{A}_{\mathrm{V}}, M_{*}$ and SSFR for Sample C galaxies $\left(i<60^{\circ}\right)$.

ation influences the survival of $\mathrm{Na} \mathrm{I}$ in the clouds.

A similar analysis applied to the outflow velocity $\left(v_{\text {off }}\right)$ instead of the EW does not yield any insight into the dominant physical parameter determining the outflow speed. We note that the dynamic range in $v_{\text {off }}$ is very small (120 - 160 $\mathrm{km} \mathrm{s}^{-1}$ ), and this makes identification of trends particularly challenging. It is also important to keep in mind that the range in galaxy physical properties probed by our study is quite small - typically of order 1 dex. For instance, we are looking for trends over a range in stellar mass of $\log \left(M_{*} / M_{\odot}\right)=$ $10.3-11.3$, whereas the full galaxy population spans over six orders of magnitude in stellar mass. This limitation is imposed by our need to study objects where $\mathrm{Na} \mathrm{D}$ absorption is common (Fig. 4).

In spite of these limitations, we can use our results to investigate a basic physical model of the outflow. Following Martin \& Bouché (2009) we suggest that the velocity of the line centroid reflects the speed of the swept-up shell of interstellar gas at the point where it blows out of the disk. Combining equations 3 and 6 from Strickland et al. (2004), for the velocity of a shell of gas at blow-out, we find $v_{\text {off }} \propto\left(\Sigma_{\mathrm{SFR}} / \rho_{0}\right)^{1 / 3}$ where $\rho_{0}$ is the gas density. Assuming $\rho_{0} \propto \Sigma_{\text {gas }} / H_{z}$, where $H_{z}$ is the scale height of the disk, and $\Sigma_{\text {gas }} \propto \Sigma_{\text {SFR }}^{1 / 1.4}$ Kennicutt $1998 \mathrm{~b})$, we find $v_{\text {off }} \propto \Sigma_{\mathrm{SFR}}^{0.1} H_{z}^{1 / 3}$. This is consistent with the very shallow scaling we see between outflow velocity and $\Sigma_{\text {SFR. }}$

Once the cool entrained gas has escaped the disk, it may be accelerated further by the ram pressure of the hot wind, or by radiation pressure on dust grains. This halo gas likely contributes to the wings of the line profile (Martin \& Bouche 2009). In Figure 16, we examine the physical drivers of the line width of the $\mathrm{Na} \mathrm{D}$ outflow component. We see that the line width is sensitive to all three parameters: $M_{*}, \Sigma_{\mathrm{SFR}}$, and $A_{V}$. The latter two are intuitive: cloud acceleration is likely related to $\Sigma_{\text {SFR }}$, while dust attenuation is important to keep the gas neutral. The positive correlation between line width and stellar mass is more puzzling. It may be that clouds with velocities above the halo escape velocity quickly dissipate after leaving the pressure-confining hot medium of the halo. The larger line widths in more massive galaxies may therefore reflect the larger size of the hot halo. 

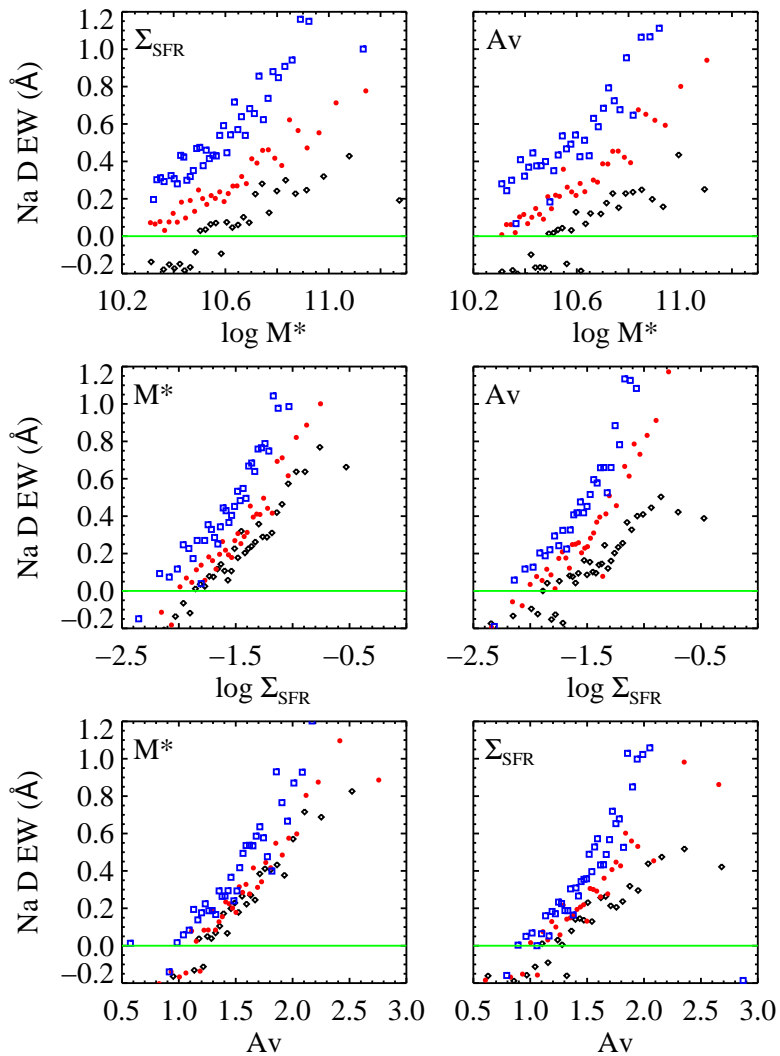

FIG. 14.- The dependence of the Na D EW of the systemic component on galaxy physical properties. In each panel, the sample is divided into three sub-bins by the parameter marked in the top-left corner. The black, red, and blue points indicate the low, medium, and high sub-bins respectively. This approach is designed to help mitigate the effects of correlations among the galaxy physical parameters (see $\$ 4.3$ for details). The physical parameters that play the dominant role in driving the trends will show the smallest offsets between the black, red, and blue points.

\subsection{Comparison with Previous Work}

A correlation between outflow velocity and galaxy SFR and rotation speed was identified by Rupke et al. (2005b) and Martin (2005) in starburst galaxies. In Figure 17we compare our data (cyan crosses) to these results. For consistency with the literature data, we use global (i.e., aperture-corrected) SFRs. Green asterisks show 1 Jy ULIRGs from Rupke et al. (2002); red squares indicate ULIRGs from Martin (2005); blue circles are LIRGs from Heckman et al. (2000); pink diamonds are $z \sim 1.4$ galaxies from (Weiner et al.|2009); and black triangles are dwarf starbursts from Schwartz \& Martin (2004). All the data are corrected to a Kroupa IMF.

The velocities of our composite spectra show good agreement with the velocities derived from individual galaxies in the same SFR range (Heckman et al. 2000; Schwartz \& Martin 2004). However, the trend with SFR is weaker than the correlation suggested by Martin (2005, black line). We note that slope of this correlation is strongly influenced by three dwarf galaxies with very low SFRs. The large scatter on $v_{\text {off }}$ at fixed SFR for the individual measurements may be due both to intrinsic variations and inclination effects.

Based on a sample of high stellar mass galaxies between $z \sim 1.4$ and $z \sim 1$, Rubin et al. (2009) suggest that outflow
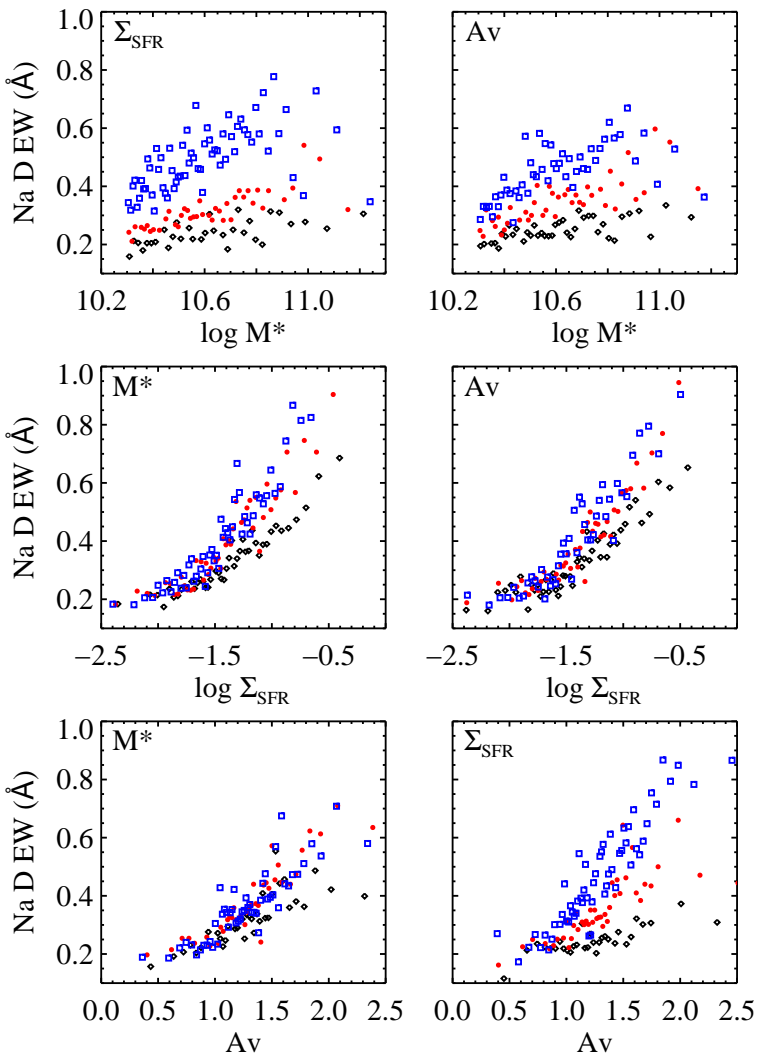

FIG. 15.- The dependence of the Na D EW of the outflow component on galaxy physical properties. As in Figure 14 the sample in each panel is divided into three sub-bins by the parameter marked in the top-left corner (black=low, red=medium, blue=high).

absorption strength (measured from $\mathrm{Mg}$ II $\lambda \lambda 2796,2803$ ) is more closely associated with SFR than with $M_{*}$, and it does not increase with increasing SSFR. Although they have not detected a strong dependence of outflow absorption strength on $\Sigma_{\mathrm{SFR}}$, they have by no means ruled it out due to the spectrum S/N limit.

\section{SUMMARY}

In this paper, we study interstellar Na I "D” $\lambda \lambda 5890,5896$ absorption in a large sample of star forming galaxies drawn from SDSS DR7. At the low SFRs probed by our sample, cool stars with prominent stellar $\mathrm{Na} \mathrm{D}$ lines make a significant contribution to the integrated light. We account for this by modeling the full galaxy continuum using the CB08 stellar population synthesis models. We find a high incidence of strong ISM Na D absorption lines $(\mathrm{EW}>0.8 \AA)$ in galaxies that are massive, heavily dust attenuated, and that have high $\Sigma_{\text {SFR }}$ (Fig. 4.)

We use the spectral stacking technique to increase the signal-to-noise ratio of the spectra and the completeness of the sample and stack spectra in various bins of galaxy physical properties. In the continuum-normalized spectra we identify two interstellar $\mathrm{Na} \mathrm{D}$ absorption components - one at the systemic velocity, which is most pronounced in edge-on galaxies, and one outflow (blue-shifted) component which is dominant in face-on galaxies. We use two-component absorp- 

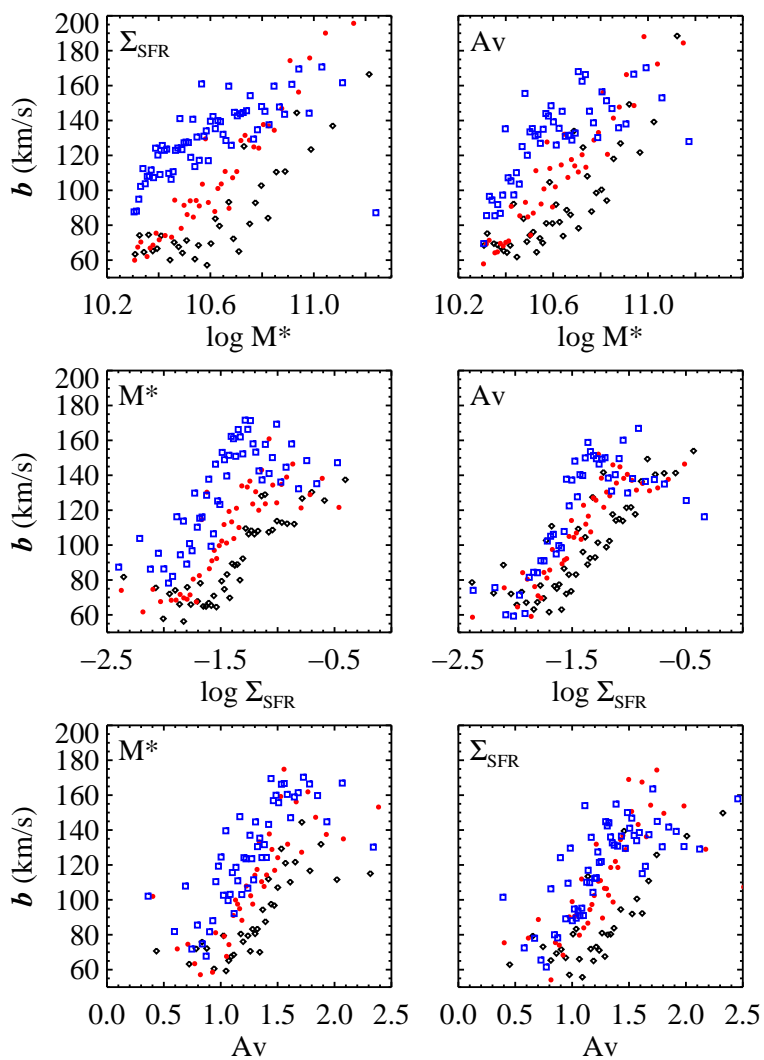

FIG. 16. - The dependence of the line width of the outflow component on galaxy physical properties. As in Figure 14 the sample in each panel is divided into three sub-bins by the parameter marked in the top-left corner (black=low, red=medium, blue=high).

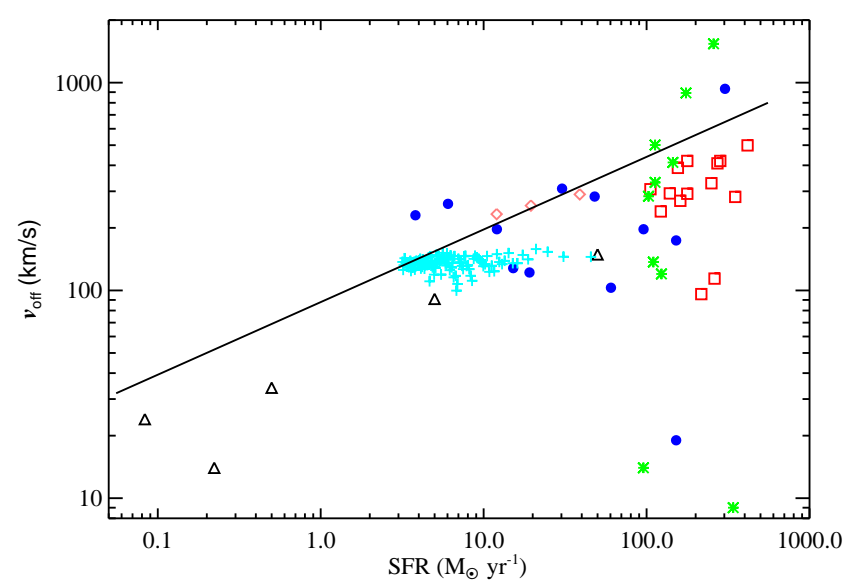

FIG. 17. - Na D outflow velocity vs. galactic SFR. Our data is shown as cyan crosses; green asterisks are 1 Jy ULIRGs from Rupke et al. (2002); red squares are ULIRGs from Martin (2005); blue circles are LIRGs from Heckman et al. (2000); black triangles are dwarf starbursts from Schwartz \& Martin (2004), and the pink diamonds are from Weiner et al. (2009). The black line is the upper envelope fit given by Martin (2005). tion line profile fits to measure the outflow velocity, the absorption line EW, the line width, covering factor, and the optical depth. As highlighted in the appendix, our results are robust with respect to the modeling of the stellar continuum. We find that:

- The ISM Na D absorption arises from cool gas in the disk, and from cool gas entrained in a galactic wind that is perpendicular to the disk and has an opening angle of $\sim 60^{\circ}$.

- For the systemic (disk) component, the Na D EW depends most strongly on the dust attenuation. Dust attenuation is undoubtedly important in shielding $\mathrm{Na} \mathrm{I}$ from the ionizing radiation of $\mathrm{OB}$ stars. At fixed $\mathrm{A}_{\mathrm{V}}$, there is an additional dependence on $\Sigma_{\mathrm{SFR}}$. Galaxies with higher $\Sigma_{\text {SFR }}$ probably have a higher filling factor of cold clouds and thus a higher $\mathrm{Na} \mathrm{D}$ covering fraction.

- For the outflow component the $\mathrm{Na}$ D EW depends strongly on $\Sigma_{\mathrm{SFR}}$ and secondarily on $\mathrm{A}_{\mathrm{V}}$. We hypothesize that the star formation surface density determines the amount of material lofted above the disk, and the dust attenuation influences the survival of $\mathrm{Na} \mathrm{I}$ in the clouds.

- The covering factor of $\mathrm{Na} \mathrm{I}$ in the outflow increases with increasing dust attenuation while the optical depth of the absorption decreases. This suggests that $\mathrm{Na} \mathrm{D}$ is able to survive in clouds with lower column density as the dust-to-gas ratio increases.

- The outflow velocity (line centroid) does not depend strongly on any galaxy physical parameters over the limited dynamic range in physical properties probed by our study. There is some evidence for a very shallow trend, $v_{\text {off }} \propto \Sigma_{\text {SFR }}^{0.1}$, which is consistent with theoretical expectations for the velocity of a swept-up shell of gas at the point where it blows out of the disk.

- The line width of the outflow component is sensitive to $\Sigma_{\mathrm{SFR}}, \mathrm{A}_{\mathrm{V}}$, and $M_{*}$. These parameters are likely to influence the acceleration of halo clouds (by ram or radiation pressure), the ability of clouds to remain neutral, and the length of time a cloud can remain pressure confined.

The question of how galactic scale outflows are influenced by AGNs is also of great interest. In a companion paper, we will study the $\mathrm{Na} \mathrm{D}$ absorption properties in SDSS galaxies hosting type 2 AGNs using methods similar to those described here.

The Na D ISM absorption line provides a convenient probe of galactic winds due to its location in the optical. However, the low EW width of the line in typical galaxy spectra and the strength of the $\mathrm{Na} \mathrm{D}$ stellar feature make interstellar $\mathrm{Na} \mathrm{D}$ absorption a challenge to measure accurately. The strong sensitivity of $\mathrm{Na}$ I to ionization by $\mathrm{OB}$ stars is also a very serious concern, in particular for studies that aim to compute mass outflow rates from $\mathrm{Na}$ I column densities and velocities. Higher redshift studies that make use of higher ionization transitions such as Mg II $\lambda 2796,2803$ may ultimately be able to provide more robust constraints over a larger range in galaxy physical properties.

Theoretical models of galaxy evolution have begun to incorporate galactic winds, using various ad-hoc prescriptions 
based on our knowledge of the cool gas. However, the majority the energy and newly-synthesized metals in the outflow resides in the hot X-ray emitting phase of the wind (D. K. Strickland \& D. C. Dinge, 2010, in prep.). To truly understand the impact of galactic winds on the evolution of galaxies and the intergalactic medium, it is crucial to measure the chemical composition and velocity of the hot gas. This will require a high sensitivity $X$-ray imaging spectrometer such as the planned International $X$-ray Observatory.

We are very grateful to the referee for useful suggestions. C.A.T thanks the Alexander von Humboldt foundation for their generous support while much of this work was completed.

Funding for the SDSS and SDSS-II has been provided by the Alfred P. Sloan Foundation, the Participating Institutions, the National Science Foundation, the U.S. Department of Energy, the National Aeronautics and Space Administration, the Japanese Monbukagakusho, the Max Planck Society, and the
Higher Education Funding Council for England. The SDSS Web Site is http://www.sdss.org/.

The SDSS is managed by the Astrophysical Research Consortium for the Participating Institutions. The Participating Institutions are the American Museum of Natural History, Astrophysical Institute Potsdam, University of Basel, University of Cambridge, Case Western Reserve University, University of Chicago, Drexel University, Fermilab, the Institute for Advanced Study, the Japan Participation Group, Johns Hopkins University, the Joint Institute for Nuclear Astrophysics, the Kavli Institute for Particle Astrophysics and Cosmology, the Korean Scientist Group, the Chinese Academy of Sciences (LAMOST), Los Alamos National Laboratory, the Max-Planck-Institute for Astronomy (MPIA), the MaxPlanck-Institute for Astrophysics (MPA), New Mexico State University, Ohio State University, University of Pittsburgh, University of Portsmouth, Princeton University, the United States Naval Observatory, and the University of Washington.

\section{REFERENCES}

Abazajian K., Adelman-McCarthy J. K., Agüeros M. A., Allam S. S., Anderson K., Anderson S. F., Annis J., Bahcall N. A., et al., 2004, AJ, 128,502

Abazajian K. N., Adelman-McCarthy J. K., Agüeros M. A., Allam S. S., Allende Prieto C., An D., Anderson K. S. J., Anderson S. F., et al., 2009, ApJS, 182, 543

Adelman-McCarthy J. K., Agüeros M. A., Allam S. S., Allende Prieto C., Anderson K. S. J., Anderson S. F., Annis J., Bahcall N. A., et al., 2008, ApJS, 175, 297

Benson A. J., Bower R. G., Frenk C. S., Lacey C. G., Baugh C. M., Cole S., 2003, ApJ, 599, 38

Bica E., Pastoriza M. G., da Silva L. A. L., Dottori H., Maia M., 1991, AJ, 102,1702

Bigiel F., Leroy A., Walter F., Brinks E., de Blok W. J. G., Madore B., Thornley M. D., 2008, AJ, 136, 2846

Bregman J. N., 1980, ApJ, 236, 577

Brinchmann J., Charlot S., White S. D. M., Tremonti C., Kauffmann G., Heckman T., Brinkmann J., 2004, MNRAS, 351, 1151

Calzetti D., Kinney A. L., Storchi-Bergmann T., 1994, ApJ, 429, 582

Cardelli J. A., Clayton G. C., Mathis J. S., 1989, ApJ, 345, 245

Chevalier R. A., Clegg A. W., 1985, Nature, 317, 44

Cole S., Lacey C. G., Baugh C. M., Frenk C. S., 2000, MNRAS, 319, 168

Dahlem M., Weaver K. A., Heckman T. M., 1998, ApJS, 118, 401

Dalcanton J. J., 2007, ApJ, 658, 941

de Vaucouleurs G., 1948, Annales d'Astrophysique, 11, 247

Engelbracht C. W., Kundurthy P., Gordon K. D., Rieke G. H., Kennicutt R. C., Smith J., Regan M. W., Makovoz D., et al., 2006, ApJ, 642, L127

Fujita A., Martin C. L., Low M., New K. C. B., Weaver R., 2009, ApJ, 698, 693

Fukugita M., Ichikawa T., Gunn J. E., Doi M., Shimasaku K., Schneider D. P., 1996, AJ, 111, 1748

Garnett D. R., 2002, ApJ, 581, 1019

González Delgado R. M., Cerviño M., Martins L. P., Leitherer C., Hauschildt P. H., 2005, MNRAS, 357, 945

Gunn J. E., Carr M., Rockosi C., Sekiguchi M., Berry K., Elms B., de Haas E., Ivezić Ž., et al., 1998, AJ, 116, 3040

Heckman T. M., 1980, A\&A, 87, 142

Heckman T. M., 2002, in J. S. Mulchaey \& J. T. Stocke ed., Extragalactic Gas at Low Redshift Vol. 254 of Astronomical Society of the Pacific Conference Series, Galactic Superwinds Circa 2001. pp 292-+

Heckman T. M., Armus L., Miley G. K., 1990, ApJS, 74, 833

Heckman T. M., Lehnert M. D., Strickland D. K., Armus L., 2000, ApJS, 129,493

Hopkins P. F., Hernquist L., Cox T. J., Robertson B., Springel V., 2006, ApJS, 163, 50

Jacoby G. H., Hunter D. A., Christian C. A., 1984, ApJS, 56, 257

Jenkins E. B., 1986, ApJ, 304, 739

Kahn F. D., 1981, in F. D. Kahn ed., Investigating the Universe Vol. 91 of Astrophysics and Space Science Library, Dynamics of the galactic fountain. pp 1-28

Kauffmann G., Heckman T. M., White S. D. M., Charlot S., Tremonti C., Brinchmann J., Bruzual G., Peng E. W., et al., 2003a, MNRAS, 341, 33

Kauffmann G., Heckman T. M., White S. D. M., Charlot S., Tremonti C., Peng E. W., Seibert M., Brinkmann J., et al., 2003b, MNRAS, 341, 54

Kauffmann G., Heckman T. M., Tremonti C., Brinchmann J., Charlot S., White S. D. M., Ridgway S. E., Brinkmann J., et al., 2003c, MNRAS, 346,1055
Kennicutt Jr. R. C., 1998a, ARA\&A, 36, 189

Kennicutt Jr. R. C., 1998b, ApJ, 498, 541

Kroupa P., 2001, MNRAS, 322, 231

Lehnert M. D., Heckman T. M., 1996, ApJ, 462, 651

Lupton R., Gunn J. E., Ivezić Z., Knapp G. R., Kent S., 2001, in

F. R. Harnden Jr., F. A. Primini, \& H. E. Payne ed., Astronomical Data Analysis Software and Systems X Vol. 238 of Astronomical Society of the Pacific Conference Series, The SDSS Imaging Pipelines. pp 269-+

Martin C. L., 2005, ApJ, 621, 227

Martin C. L., 2006, ApJ, 647, 222

Martin C. L., Bouché N., 2009, ApJ, 703, 1394

Martin C. L., Kobulnicky H. A., Heckman T. M., 2002, ApJ, 574, 663

Ménard B., Wild V., Nestor D., Quider A., Zibetti S., 2009, ArXiv: 0912.3263

Murray N., Martin C. L., Quataert E., Thompson T. A., 2007, ApJ, 660, 211

Murray N., Quataert E., Thompson T. A., 2005, ApJ, 618, 569

Mutchler M., Bond H. E., Christian C. A., Frattare L. M., Hamilton F., Januszewski W., Levay Z. G., Mountain M., et al., 2007, PASP, 119,

Ohyama Y., Taniguchi Y., Iye M., Yoshida M., Sekiguchi K., Takata T., Saito Y., Kawabata K. S. et al., 2002, PASJ, 54, 891

Oppenheimer B. D., Davé R., Kereš D., Fardal M., Katz N., Kollmeier J. A., Weinberg D. H., 2009, ArXiv: 0912.0519

Osterbrock D. E., Ferland G. J., 2006, Astrophysics of gaseous nebulae and active galactic nuclei

Padilla N. D., Strauss M. A., 2008, MNRAS, 388, 1321

Pettini M., Rix S. A., Steidel C. C., Adelberger K. L., Hunt M. P., Shapley A. E., 2002, ApJ, 569, 742

Pettini M., Steidel C. C., Adelberger K. L., Dickinson M., Giavalisco M., 2000, ApJ, 528, 96

Phillips A. C., 1993, AJ, 105, 486

Rubin K. H. R., Weiner B. J., Koo D. C., Martin C. L., Prochaska J. X., Coil A. L., Newman J. A., 2009, ArXiv: 0912.2343

Rupke D. S., Veilleux S., Sanders D. B., 2002, ApJ, 570, 588

Rupke D. S., Veilleux S., Sanders D. B., 2005a, ApJS, 160, 87

Rupke D. S., Veilleux S., Sanders D. B., 2005b, ApJS, 160, 115

Salim S., Rich R. M., Charlot S., Brinchmann J., Johnson B. D.,

Schiminovich D., Seibert M., Mallery R., et al., 2007, ApJS, 173, 267

Sánchez-Blázquez P., Peletier R. F., Jiménez-Vicente J., Cardiel N., Cenarro A. J., Falcón-Barroso J., Gorgas J., Selam S., et al., 2006, MNRAS, 371, 703

Schlegel D. J., Finkbeiner D. P., Davis M., 1998, ApJ, 500, 525

Schwartz C. M., Martin C. L., 2004, ApJ, 610, 201

Shapiro P. R., Field G. B., 1976, ApJ, 205, 762

Shapley A. E., Steidel C. C., Pettini M., Adelberger K. L., 2003, ApJ, 588, 65

Silk J., 2003, MNRAS, 343, 249

Silk J., Rees M. J., 1998, A\&A, 331, L1

Smith J. A., Tucker D. L., Kent S., Richmond M. W., Fukugita M., Ichikawa T., Ichikawa S., Jorgensen A. M., et al., 2002, AJ, 123, 2121

Strauss M. A., Weinberg D. H., Lupton R. H., Narayanan V. K., Annis J., Bernardi M., Blanton M., Burles S., 2002, AJ, 124, 1810

Strickland D. K., Heckman T. M., Colbert E. J. M., Hoopes C. G., Weaver K. A., 2004, ApJ, 606, 829

Stringer M. J., Benson A. J., Bundy K., Ellis R. S., Quetin E. L., 2009, MNRAS, 393, 1127

Tremonti C. A., Heckman T. M., Kauffmann G., Brinchmann J., Charlot S., White S. D. M., Seibert M., Peng E. W., et al., 2004, ApJ, 613, 898 


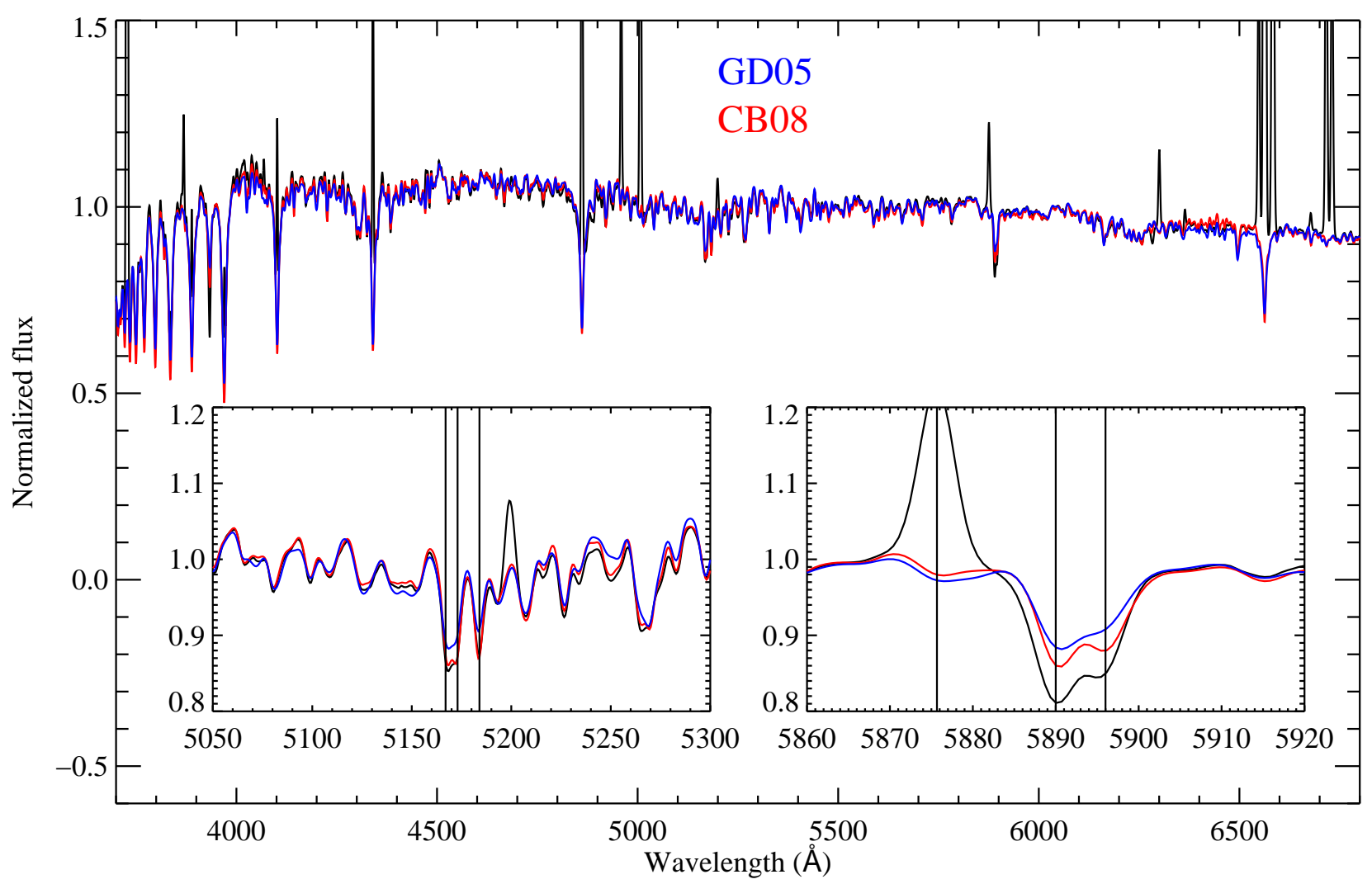

FIG. 18. - This figure is the same as Figure 5 but we add the GD05 stellar continuum model (blue line) for comparison. The CB08 model provides a better fit to the stellar population.

Tremonti C. A., Moustakas J., Diamond-Stanic A. M., 2007, ApJ, 663, L77

Veilleux S., Cecil G., Bland-Hawthorn J., 2005, ARA\&A, 43, 769

Weiner B. J., Coil A. L., Prochaska J. X., Newman J. A., Cooper M. C.,

Bundy K., Conselice C. J., Dutton A. A., et al., 2009, ApJ, 692, 187

Worthey G., Faber S. M., Gonzalez J. J., Burstein D., 1994, ApJS, 94, 687
York D. G., Adelman J., Anderson Jr. J. E., Anderson S. F., Annis J., Bahcall N. A., Bakken J. A., Barkhouser R., et al., 2000, AJ, 120, 1579

\section{APPENDIX}

Here we compare the stellar continuum of González Delgado et al. (2005, hereafter GD05) and CB08 stellar population models to check whether our results are model dependent.

In $\S 2.1$, we noted that stellar $\mathrm{Na} \mathrm{D}$ appears to be over-estimated in our continuum fits, resulting in emission at the systemic velocity in the continuum-normalized spectra. The libraries of stellar spectra used by CB08 are empirical, so Na D could be contaminated by Milky Way absorption. One important question is to what extent our results are affected by the stellar population models used to estimate the continuum. In order to answer this question, we repeat all our analysis using the models of GD05 which use fully theoretical stellar libraries. Figure 18 shows both CB08 and GB05 model fits to the stacked spectrum shown in Figure 5 The CB08 model is shown in red and the GD05 model in blue. The CB08 model clearly provides a better fit of the the full spectrum and the region around the $\mathrm{Mg}$ I triplet.

Using the GD05 theoretical models, all the trends shown in Figs. 9, 12, and 13 remain. On average, the outflow velocity decreases by $\sim 40-60 \mathrm{~km} \mathrm{~s}^{-1}$; the Na D EW increases by $\sim 0.3 \AA$, for both disk-like and outflow components; and the covering factors for the two components increase by $10 \%$. The line widths for the systematic component are the same as that from CB08, since they are bound to He I. The width of outflow component increases by $\sim 25-30 \mathrm{~km} \mathrm{~s}^{-1}$ and more scatter is introduced in the trends with galaxy physical properties. The largest change caused by the using different stellar population models is the optical depth $\tau_{0}$. However, this parameter is not very strongly constrained in either case. In summary, the CB08 models provide a better overall fit to the stellar continuum. However, our basic results are not sensitive to the stellar population models used. 